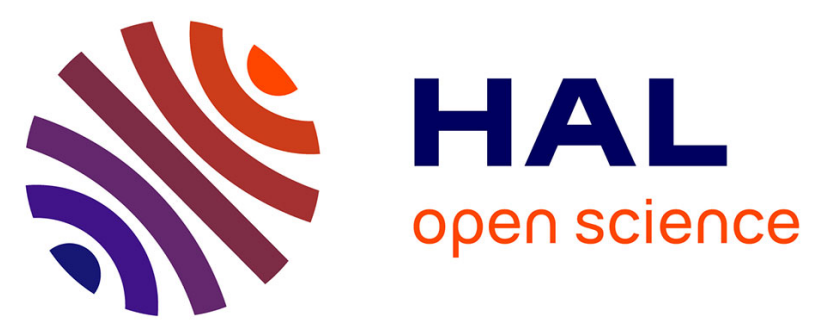

\title{
Interplanetary hydrogen absolute ionization rates: Retrieving the solar wind mass flux latitude and cycle dependence with SWAN/SOHO maps
}

Eric Quémerais, Rosine Lallement, Stéphane Ferron, Dimitra Koutroumpa, Jean-Loup Bertaux, Erkki Kyrölä, Walter Schmidt

\section{To cite this version:}

Eric Quémerais, Rosine Lallement, Stéphane Ferron, Dimitra Koutroumpa, Jean-Loup Bertaux, et al.. Interplanetary hydrogen absolute ionization rates: Retrieving the solar wind mass flux latitude and cycle dependence with SWAN/SOHO maps. Journal of Geophysical Research Space Physics, 2006, 111, pp.A09114. 10.1029/2006JA011711 . hal-00186451

\section{HAL Id: hal-00186451 https://hal.science/hal-00186451}

Submitted on 9 Oct 2020

HAL is a multi-disciplinary open access archive for the deposit and dissemination of scientific research documents, whether they are published or not. The documents may come from teaching and research institutions in France or abroad, or from public or private research centers.
L'archive ouverte pluridisciplinaire HAL, est destinée au dépôt et à la diffusion de documents scientifiques de niveau recherche, publiés ou non, émanant des établissements d'enseignement et de recherche français ou étrangers, des laboratoires publics ou privés. 


\title{
Interplanetary hydrogen absolute ionization rates: Retrieving the solar wind mass flux latitude and cycle dependence with SWAN/SOHO maps
}

\author{
Eric Quémerais, ${ }^{1}$ Rosine Lallement, ${ }^{1}$ Stéphane Ferron, ${ }^{1}$ Dimitra Koutroumpa, ${ }^{1}$ \\ Jean-Loup Bertaux, ${ }^{1}$ Erkki Kyrölä, ${ }^{2}$ and Walter Schmidt ${ }^{2}$ \\ Received 8 March 2006; revised 26 May 2006; accepted 15 June 2006; published 28 September 2006.
}

[1] We present results of the total hydrogen ionization rate obtained from the inversion of almost 10 years of full-sky maps of the interplanetary Lyman $\alpha$ background measured by the SWAN instrument on SOHO. Thanks to a new estimate of the absolute calibration of the SWAN instrument and its variation during the 10 years of operation of SOHO, we are able to derive absolute values of the ionization rate as well as its latitudinal dependence. We show how the anisotropy of the ionization rate changes from solar minimum to solar maximum. At solar maximum, the so-called ionization groove has completely disappeared and the ionizing fluxes are the same at all heliographic latitudes. We find that the hydrogen ionization cavity which surrounds the Sun increases in size with solar activity. This is evidenced by the low IP Lyman $\alpha$ intensities measured during and after the solar maximum. Our model calculation also shows that the increased radiation pressure is not sufficient to explain the larger cavity observed at solar maximum. We find also that ionization rates derived from in situ solar wind measurements do agree with the SWAN results at solar minimum but are significantly smaller at solar maximum. Derived in situ ionization rates do not show the solar cycle dependence we see from the SWAN data. In conclusion, we discuss possible explanations for this discrepancy.

Citation: Quémerais, E., R. Lallement, S. Ferron, D. Koutroumpa, J.-L. Bertaux, E. Kyrölä, and W. Schmidt (2006), Interplanetary hydrogen absolute ionization rates: Retrieving the solar wind mass flux latitude and cycle dependence with SWAN/SOHO maps, J. Geophys. Res., 111, A09114, doi:10.1029/2006JA011711.

\section{Introduction}

[2] The interplanetary Lyman $\alpha$ glow was first observed in the late 1960s [Bertaux and Blamont, 1971; Thomas and Krassa, 1971]. This diffuse emission seen in all directions of the sky is caused by the scattering of solar Lyman $\alpha$ photons by hydrogen atoms present in the interplanetary medium. These hydrogen atoms penetrate inside the heliosphere because of the motion of the solar system relative to the local interstellar medium. This creates the so-called interstellar wind.

[3] In the vicinity of the Sun, the interplanetary hydrogen atoms can either be ionized by charge exchange with solar wind protons or photoionized by EUV photons. These ionization processes combined with the dynamical effects of the Sun on the atom trajectories create a cavity void of neutral hydrogen atoms close to the Sun. This cavity is elongated in the downwind direction, i.e., in the opposite direction to the incoming interstellar wind.

\footnotetext{
${ }^{1}$ Service d'Aéronomie, Verrières le Buisson, France.

${ }^{2}$ Finnish Meteorological Institute, Helsinki, Finland.
}

Copyright 2006 by the American Geophysical Union. 0148-0227/06/2006JA011711
[4] It has been shown through modeling by Joselyn and Holzer [1975] that if the ionizing fluxes from the Sun are not isotropic, the spatial distribution of atomic hydrogen atoms in the interplanetary medium would be greatly affected. The authors pointed out that this effect could be observed by remote sensing with a mapping of the interplanetary Lyman $\alpha$ emission.

[5] Kumar and Broadfoot [1979], analyzing observations made at the end of 1973 by the Mariner 10 UV spectrophotometer, showed that the data were better fitted when using an anisotropic ionizing flux from the Sun. They found that the ionization rate was smaller at the poles of the Sun. Work by Witt et al. [1979] based on the same data set estimated that the ionization rate decreased by approximately $25 \%$ from the equator to the poles.

[6] Lallement et al. [1985] analyzed the interplanetary Lyman $\alpha$ data recorded by the Prognoz 5/6 Lyman $\alpha$ photometers in 1976 and 1977. They showed that the Lyman $\alpha$ intensity pattern was better represented when an anisotropic ionization flux from the Sun was assumed. Once again, the authors found that the ionization rate was smaller at the poles. The decrease of the total ionization rate from ecliptic value to pole value was equal to $40 \%$. It was concluded that the anisotropy observed at the time of the Mariner 10 observations (1973) had increased to reach the 
value observed by the Prognoz instruments in 1976 and 1977, close to the solar minimum.

[7] The SWAN instrument, which is one of the 12 instruments on board the SOHO spacecraft [Bertaux et al., 1995], has been recording the variations of the interplanetary glow from early 1996 to present, thus covering most of the last 11-year solar cycle (cycle 23). The main scientific objective of this instrument is the study of the anisotropy of the hydrogen ionization rate and its variations during the solar cycle. Launched at the end of 1995, the SOHO mission started its routines operations in early 1996 in time for the solar minimum.

[8] After almost 10 years of operations, the SWAN instrument has recorded more than 1350 maps of the full sky ( $4 \pi$ steradian) intensity pattern of the interplanetary Lyman $\alpha$ background. Early maps in 1996 clearly confirmed the existence of the groove in the intensity pattern which was previously observed in the Prognoz 5 data [Bertaux et al., 1996]. This groove appears close to the ecliptic plane and is caused by the larger hydrogen ionization rate that is associated with the slow solar wind. Near solar minimum, the slow solar wind is concentrated in the regions of low heliographic latitudes which are close to the ecliptic plane. As a result, this creates a region where ionization is enhanced and has fewer hydrogen atoms and backscattered Lyman $\alpha$ photons. This also creates two local maxima in the Lyman $\alpha$ intensity pattern, one north of the ecliptic plane and one in the south [Bertaux et al., 1997]. Early analyses of the SWAN maps were published by Summanen et al. [1997] and Bzowski et al. [2003].

[9] Maps recorded in 2001 clearly showed that the groove had disappeared, suggesting that the ionization rate becomes isotropic at the time of the solar maximum. This work presents a complete analysis of the SWAN full sky maps. Our aim is to provide absolute values of the total ionization rate of hydrogen as a function of heliographic latitude between 1996 and 2005. This is achieved by a nonlinear least squares fit of monthly averages maps (LevenbergMarquardt method).

[10] The first section presents the SWAN data that have been used in this analysis. Monthly averaged data have been used to decrease the effects of the 27-day variation of the solar Lyman $\alpha$ flux [Bertaux et al., 2000]. The absolute calibration of the instrument presented here is new to this work. It is the official calibration and should be used in future works.

[11] The second section presents the model used in the fit process. This model is based on the hot model developed by Lallement et al. [1985] with corrections for an anisotropic ionization rate and any values for the radiation pressure coefficient $\mu$. An actual solar line profile derived from the SUMER/SOHO measurements [Lemaire et al., 2002] is used. A correction for radiative transfer effects based on the work by Quémerais [2000] is also included. The numerical values of the solar illuminating flux and radiation pressure coefficient are derived from the UARS/SOLSTICE [Woods et al., 2000]. The parameters of the interstellar wind used as boundary conditions in the model computations are derived from the analysis of the SWAN hydrogen absorption cell data [Lallement et al., 2005; Koutroumpa et al., 2005].
[12] Results of the nonlinear least squares fit to the SWAN data are presented in the following section. We present temporal and latitudinal variations over the period of operation of the SWAN instrument. The final section presents future works, specifically how to separate the total ionization rate between the photoionization rate and charge exchange ionization rate. This action will be necessary for retrieving accurate values of the solar wind mass flux at all latitudes from the SWAN data set.

\section{SWAN Data: Full Sky Images From 1996 to 2005}

2.1. Data Presentation

[13] The SWAN instrument was designed to map the interplanetary background at Lyman $\alpha$. It was launched in December 1995 as part of the SOHO payload and has been recording maps of the interplanetary background since January 1996. It is still operating at present. Scientific operations were stopped between June and October 1998 following the loss of attitude control of the SOHO spacecraft in June 1998. They resumed in October 1998 after the recovery. They stopped again in January 1999 after the loss of the last gyroscope of the SOHO spacecraft. Operations of the SWAN instrument have been nearly continuous since the end of February 1999.

[14] The instrumental design is presented by Bertaux et al. [1995]. The experiment is composed of three independent units. There are two identical sensor units placed on opposite sides of the spacecraft ( $+\mathrm{Z}$ and $-\mathrm{Z}$ faces) linked to a central Digital Processing Unit also on the $-Z$ panel. Because the $+Z$ axis of the SOHO reference frame is the projection of the solar rotation axis on the plane perpendicular to the SOHO-Sun vector, the $+Z$ sensor mainly sees the north ecliptic hemisphere, whereas the $-Z$ sensor sees the south ecliptic hemisphere.

[15] The detectors are multianode Micro Channel Plate (MCP) detector tubes from Hamamatsu. A CsI photocath-

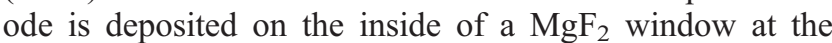
entrance of MCP. The window provides a lower cutoff at roughly $115 \mathrm{~nm}$, and the CsI photocathode (solar blind) provides the upper cutoff around $185 \mathrm{~nm}$. The Micro Channel Plate multiplier containing two stacks multiplies the photonelectron entering the tube, and the total corresponding charge at the output of the MCP is collected on a set of several square anodes. The optical layout is detailed by Bertaux et al. [1995]. The instantaneous field of view of one sensor unit is a square of $5 \times 5^{\circ}$ divided in $5 \times$ 5 pixels (anodes) of $1^{\circ}$ square each. The central line of sight can be oriented toward any point of one hemisphere by a mechanical periscope system containing two toroidal mirrors at $45^{\circ}$ incidence.

[16] In this work we have used the full sky observations of SWAN. Since January 1996, SWAN has performed more than 1350 such maps. These observations are made roughly three times per week. Each of the sensors covers roughly half of the sky in 24 hours. Individual measurements of the 25 pixels are integrated over $13 \mathrm{~s}$; then the line of sight is changed by rotating one of the motors by $2.5^{\circ}$. A new integration period begins every $15 \mathrm{~s}$, which leaves $2 \mathrm{~s}$ to move the motors to position which is quite enough for a rotation of $2.5^{\circ}$. With a total field of view of $5 \times 5$ degrees 

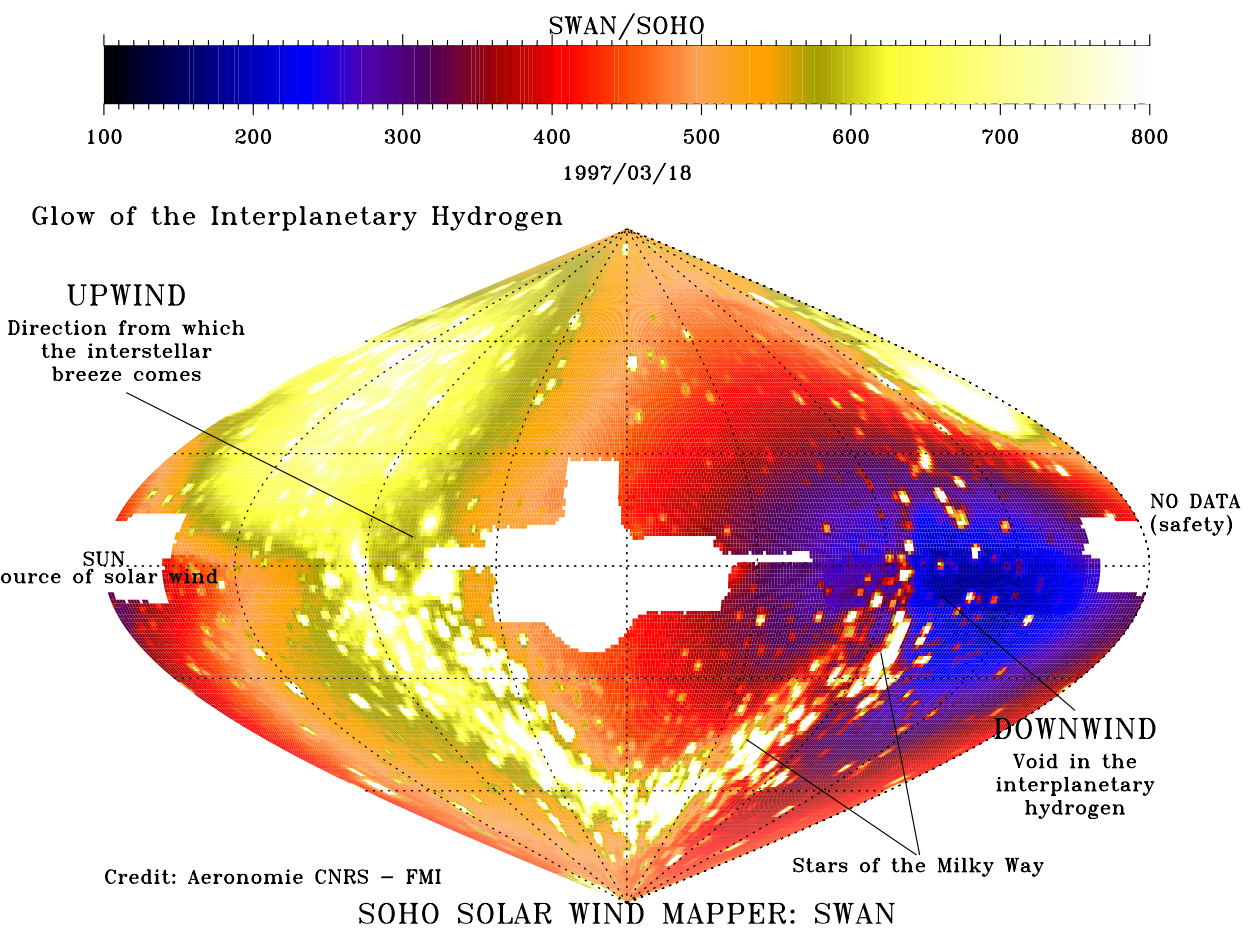

Figure 1. Full sky observation made by SWAN on 18 March 1997. The color scale is in units of counts per second for the central pixel of the $+Z$ sensor. The white dots correspond to the emission of hot stars, mostly along the plane of the Milky Way. The two masked areas correspond to the shadow of the spacecraft (center) and to the shadows of the Sun shields which protect the sensors from direct sunlight. The map is shown in ecliptic (J2000) coordinates.

and a movement of 2.5 degrees on both axes, each direction of the sky is observed at least four times by one sensor. Owing to the geometry of the periscope system [Bertaux et al., 1995], the directions close to the $+Z$ and $-Z$ axes of the spacecraft are oversampled.

[17] Both motors of the periscope system have to be rotated by $180^{\circ}$ to map the hemisphere facing the sensor unit. This means that a full sky image is composed of two halves, each one corresponding to one sensor. Each half is composed of about 5300 individual packets of 25 measurements, one for each pixel. The map is obtained by binning together all individual data in a given grid. The grid step is usually chosen equal to the individual pixel resolution, i.e., $1^{\circ}$. Prior to binning, a correction is computed to account for variations in pixel sensitivity (flat field correction). The reference pixel is always the central pixel of each array.

[18] There are two areas close to the ecliptic plane that are seen by both sensors. They are used to cross-calibrate the two sensors. The measurements of the sensor on the $-\mathrm{Z}$ side are multiplied by the cross-calibration factor. In the last step, the two half images are combined to form one image of the full sky at Lyman $\alpha$. The count rate corresponds to the actual count rate of the central pixel of the $+\mathrm{Z}$ sensor.

[19] Figure 1 shows a typical image of the Lyman alpha background measured by SWAN. This image is composed of the two halves measured by the two sensors. It has been computed for a $1 \times 1^{\circ}$ resolution grid. There are two areas in the sky that cannot be observed. The largest one (center of Figure 1) is blocked by the spacecraft. The smallest one corresponds to the combined shadows of the two Sun shields which protect the two sensors from direct sunlight.
The white dots in the figure are due chiefly to the emission of hot stars close to the galactic plane. They are very dense in the south ecliptic hemisphere for instance. Unfortunately, the chromatic aberration created by the optical layout also increases the contamination by star light. Because the refraction index of $\mathrm{MgF}_{2}$ is changing rapidly in the far UV, all wavelengths cannot be focused together on the detector. As a result, the image of a star is generally larger than one pixel, this means that the surrounding pixels will also measure a fraction of the starlight. In the areas of the sky close to the galactic plane, unresolved stellar light creates a continuous background that is very difficult to remove without a forward modeling of the instrumental response in the full range $(115-185 \mathrm{~nm})$. Moreover, the lack of actual data on the spectrum of many stars in the same range makes it impossible to actually compute the stellar background contaminating the SWAN data.

[20] To solve this problem, we have used the measurements of the so-called $\mathrm{BaF}_{2}$ pixel of the $+\mathrm{Z}$ sensor. As mentioned by Bertaux et al. [1995], each detector has two active pixels on the sides of the $5 \times 5$ array. One of the side pixels of the $+Z$ sensor has been covered by a window made with $\mathrm{BaF}_{2}$. This window is opaque for Lyman $\alpha$ photons. Using the data recorded by this pixel over many months, we have compiled a full sky map which excludes the Lyman $\alpha$ background. This map can be used to determine which areas of the sky are free from stellar contamination. In this way, we have created a mask which allows us to keep only the data uncontaminated by starlight.

[21] Figure 2 and Figure 3 show maps after removal of star light contamination. The maps are shown in ecliptic 


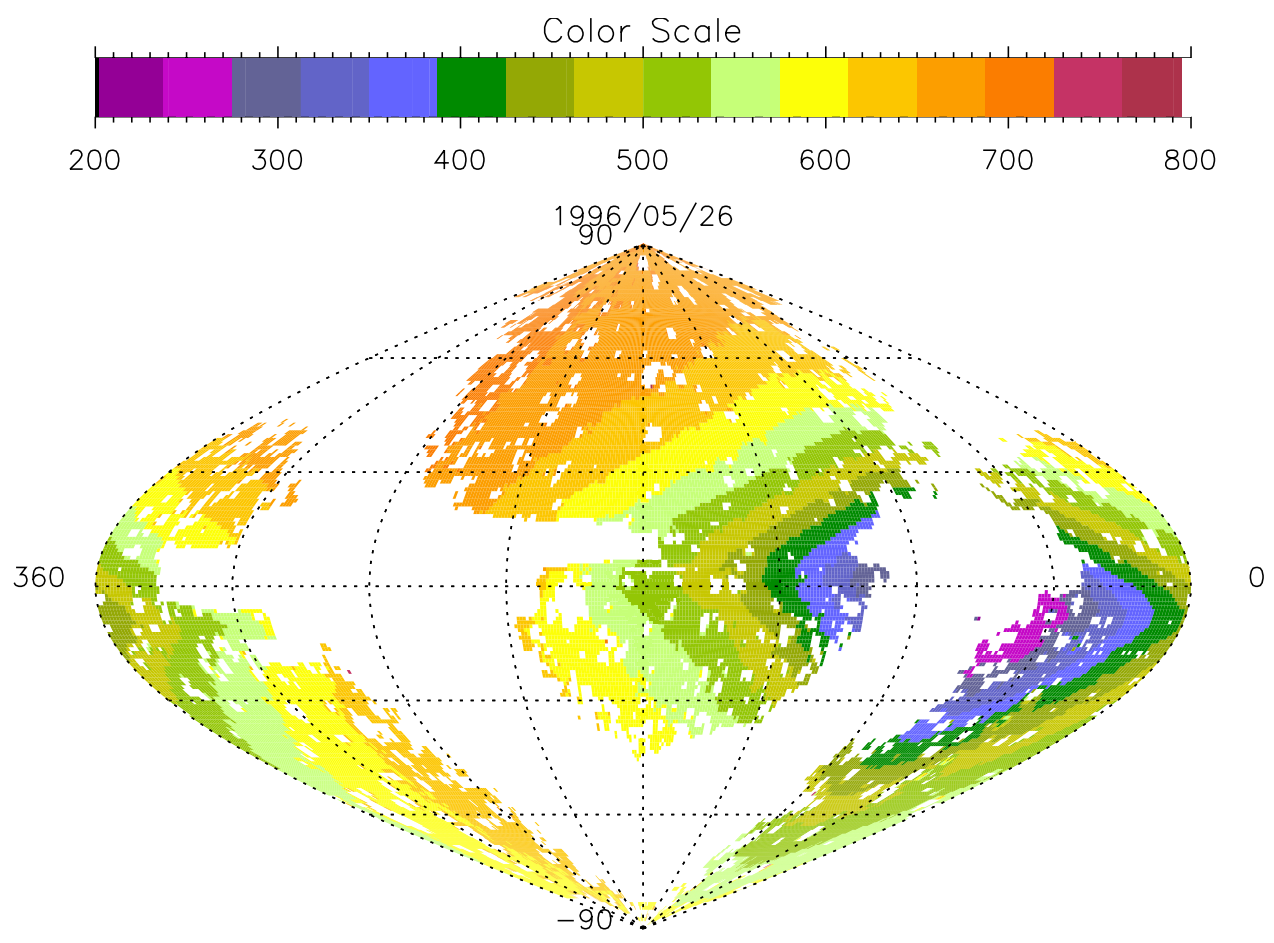

Figure 2. Example of the data used in this analysis. All areas where the count rate of the $\mathrm{BaF}_{2}$ window pixel are larger than 10 counts per second have been masked. The map is shown in ecliptic (J2000) coordinates. Regions within $20^{\circ}$ of the galactic plane have been removed. The shadows of the spacecraft and the Sun shields are superimposed on the masked areas. Both ecliptic poles are visible as well as the crosswind regions. The data are shown in counts per second.

coordinates. They have been chosen to point out the change of the intensity pattern between 1996 and 2000. The units are in counts per second for the central pixel of sensor $+\mathrm{Z}$. Almost all areas within $20^{\circ}$ of the Milky Way plane have been removed. The area of the sky masked by the spacecraft is not the same on both maps because it depends on the position of SOHO around the Sun. For Figure 3, the downwind region is very much undersampled, partly because the shadow of the spacecraft is superimposed on the masked regions. It should be noted that the data coverage between both poles is roughly uniform, and that data for all heliographic latitudes are available. Therefore the remaining data are quite sufficient to study the latitudinal variation of the total ionization rate.

\subsection{Absolute Calibration}

[22] The data used to perform this analysis are expressed in absolute units, i.e, Rayleighs. We have determined the absolute calibration of the SWAN $+Z$ sensor over all of its period of operation, i.e., from January 1996 to September 2005. As mentioned above, all count rates for the $-Z$ sensor are converted into equivalent values for the $+Z$ sensor, so only the calibration of the $+Z$ sensor is needed here.

[23] This calibration has been made by comparing the SWAN data with HST/GHRS and HST/STIS measurements of the interplanetary background [Clarke et al., 1998; J. T. Clarke, private communication, 2005]. The first spectrum was recorded in 1996 at the beginning of the SWAN operations. It was used by Quémerais and Bertaux [2002] to compute the initial calibration of the $+Z$ sensor. The second spectrum was recorded by STIS in March 2001. Between those two dates, we have determined the relative variation of the sensitivity of the $+Z$ sensor by monitoring stars and by computing the cross-calibration factor between the $+Z$ and the $-Z$ sensors when jumps occurred.

[24] The weekly monitoring of selected stars has shown that the $+Z$ sensor of SWAN has a constant sensitivity except for a series of events which caused strong degradation of the sensitivity. The relative variation of the sensitivity was determined from 1996 to 2001 by Quémerais and Bertaux [2002]. This analysis was recently extended until September 2005. During its nearly 10 years of operation, the sensitivity of the SWAN $+Z$ sensor has changed three times. The first two changes are degradations of the sensitivity due to human error. Following the loss of attitude control of SOHO in June 1998, it appeared that the $+\mathrm{Z}$ detector was hit directly by sunlight for an unknown period of time. This resulted in an overall degradation of the sensor sensitivity by roughly a factor of 2 . This value could be precisely determined because the $-Z$ sensor was not harmed. The degradation was then determined by comparing the cross calibration factor of the sensors before the loss of attitude control and after recovery. The second degradation occurred on 17 November 2000. Owing to an erroneous command sent to the instrument, the $+\mathrm{Z}$ sensor line of sight was pointed toward the spacecraft for an estimated period of 16 hours. Solar light reflected on the spacecraft caused a very large count rate (around $10^{5}$ counts per second, i.e., 3 orders of magnitude larger than the count rate of the typical observation). This resulted in a second loss of sensitivity of roughly a factor $4 / 3$. It must be noted that following the 17 November event, the sensitivity of the $+Z$ 


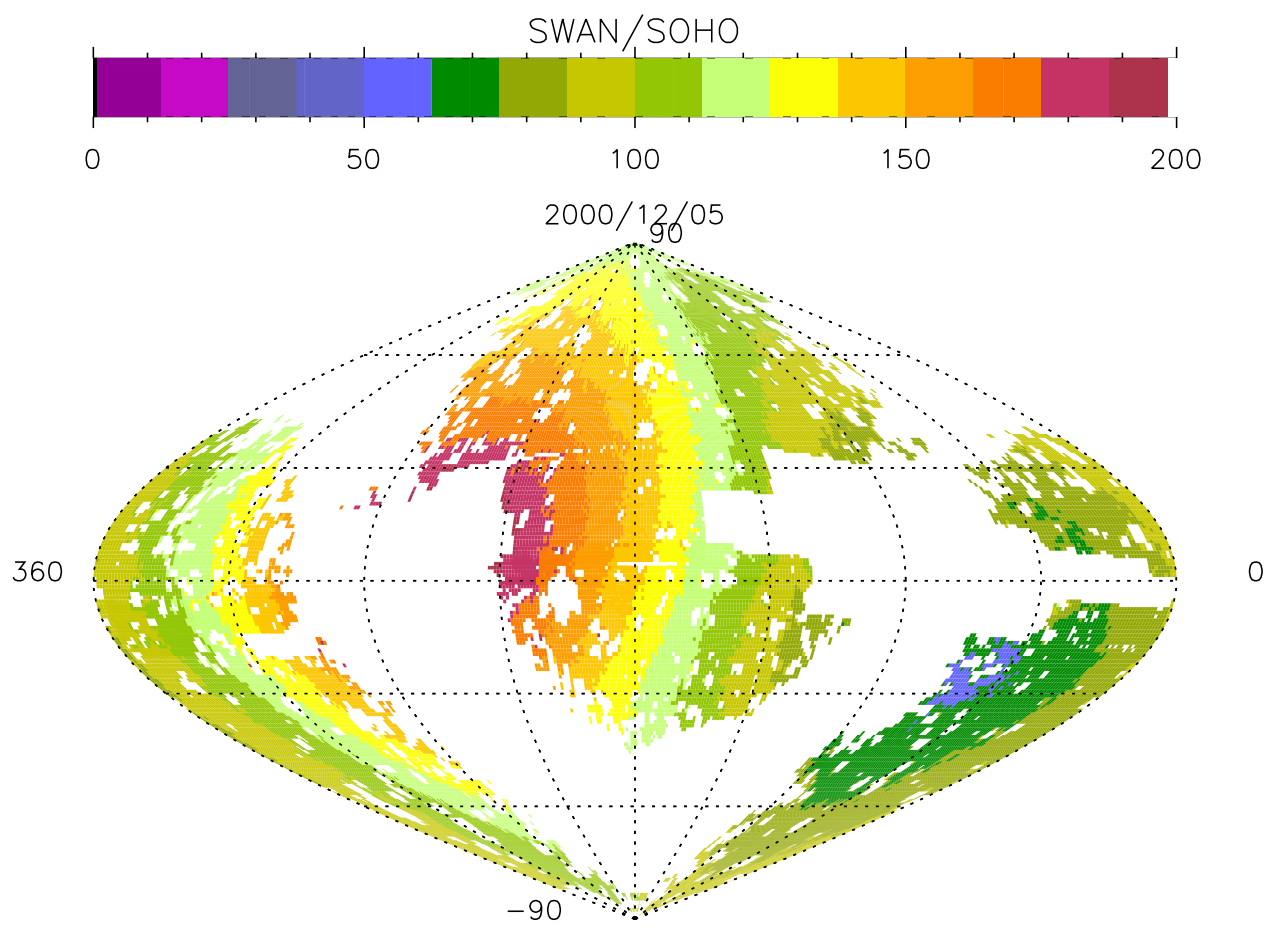

Figure 3. Second example of the data used in the analysis. All areas where the count rate of the $\mathrm{BaF}_{2}$ window pixel are larger than 10 counts per second have been masked. The map is shown in ecliptic (J2000) coordinates. Regions within $20^{\circ}$ of the galactic plane have been removed. Here the regions of the sky obstructed by the spacecraft are not the same as in the previous figure because they depend on the position of SOHO around the Sun.

sensor continued to decrease over the following week or so. It was completely stable again on 26 November. This result was found by computing the cross-calibration factor between both sensors over that period while nothing peculiar happened to the $-\mathrm{Z}$ sensor. The last change in sensitivity occurred on 26 April 2001. This change was made by increasing the high-voltage setting of the MCP to increase the gain of the intensifier. The sensitivity of the $+Z$ sensor has been constant since that date. The actual values for the sensitivity of the $+Z$ sensor are summarized in Table 1 .

[25] These values will be used in this work to express the SWAN data in absolute units. They should be used by all future works using these data and can be considered as the official SWAN calibration values.

[26] Figure 4 shows the intensity recorded by SWAN toward the upwind direction (within $10^{\circ}$ ) and the north ecliptic pole. These data are corrected for instrumental effects and are given in Rayleigh units. Clearly visible on these data are 25-day variations caused by the solar rotation. There are a few other interesting features displayed on this figure. First, contrary to our expectations, the maximum IP intensity is not observed during solar maximum but roughly 1 year before. This is due to the combination of antagonistic effects. As the illuminating Lyman $\alpha$ flux increases, the number of photons emitted by the Sun increases but the radiation pressure also increases. This depletes the inner heliosphere of hydrogen atoms. Increasing photoionization will also decrease the number of atoms. Second, the north pole intensity is very well correlated to the position of SOHO around the Sun. When SOHO is upwind from the Sun, i.e., closer to the maximum of emissivity the intensity is larger. However, this is not true for the upwind line of sight. In the first 2 years, we see an anticorrelation with the position with a maximum when the observer is downwind. Then we see a correlation in 1999 and 2000. After 2001, we do not see any clear correlation. These changes are most likely linked to the size of the ionization cavity. When the cavity is large, i.e., larger than the orbit of SOHO around the Sun, then the upwind intensity will not change much with the position of the observer. This is what happens after 2001. However, during solar minimum, the cavity is smaller than $1 \mathrm{AU}$, and then a downwind observer sees what an upwind observer sees, plus the contribution within 1 AU. This applies only to lines of sight which cross the cavity close to the Sun. This is why it shows on the upwind intensity and not on the north ecliptic intensity. These effects help us to understand how the hydrogen distribution changes in the inner heliosphere during the solar cycle.

\section{Model Description}

[27] In this section we will briefly describe the forward model used to compute the Lyman $\alpha$ intensity in the interplanetary medium.

Table 1. Calibration Factor of the $+Z$ Sensor

\begin{tabular}{cccc}
\hline Start Date & End Date & $\begin{array}{c}\text { Rayleigh Per, } \\
\text { counts per s }\end{array}$ & $\begin{array}{c}\text { High Voltage } \\
\text { Setting }\end{array}$ \\
\hline $1996 / 01 / 01$ & $1998 / 06 / 23$ & $1.190 \pm 0.120$ & $2033 . \mathrm{V}$ \\
$1998 / 10 / 20$ & $2000 / 11 / 17$ & $3.037 \pm 0.374$ & $2033 . \mathrm{V}$ \\
$2000 / 11 / 30$ & $2001 / 04 / 26$ & $3.924 \pm 0.551$ & $2033 . \mathrm{V}$ \\
$2001 / 04 / 26$ & present & $2.512 \pm 0.403$ & $2057 . \mathrm{V}$ \\
\hline
\end{tabular}




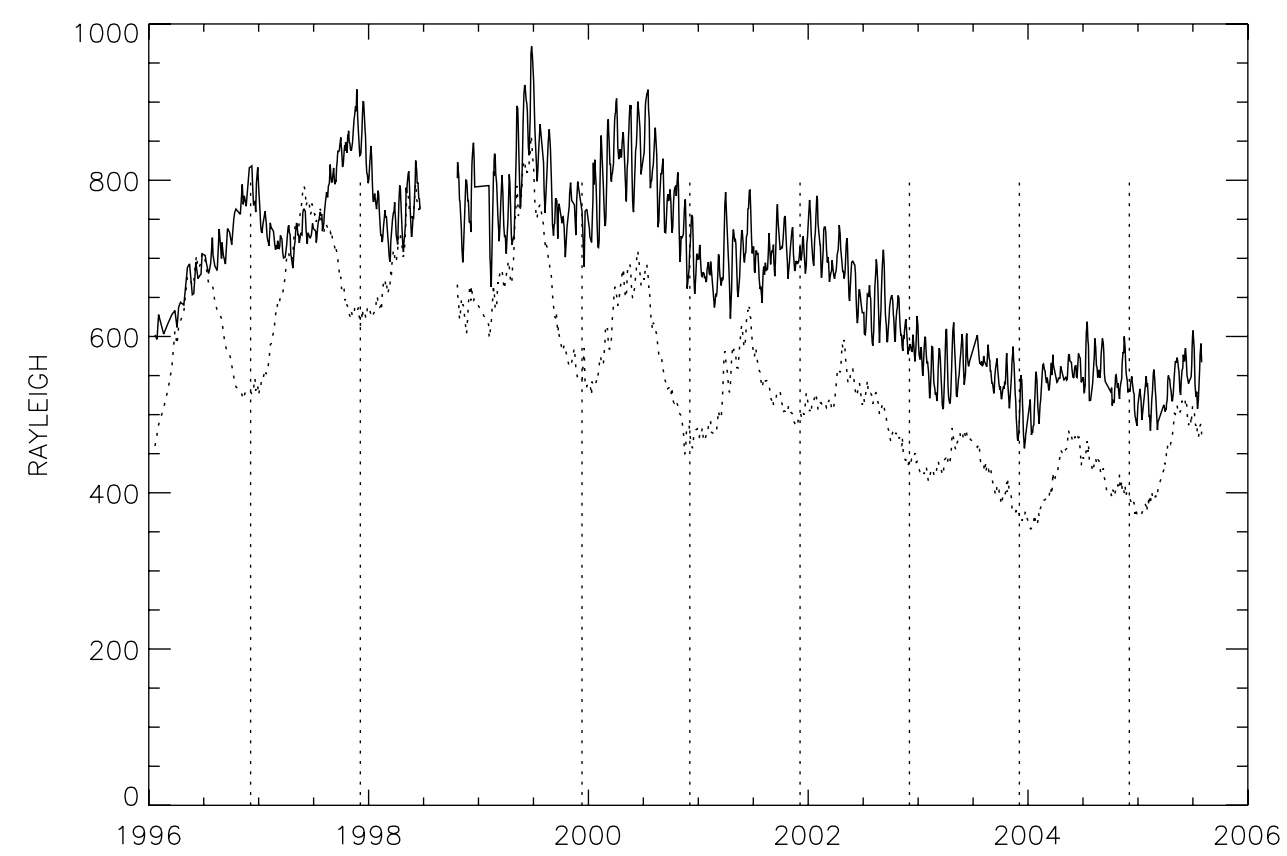

Figure 4. Plot of the intensity for two lines of sight recorded by SWAN between 1996 and 2005. The intensities are shown in Rayleigh, i.e., corrected for instrumental effects. The solid line corresponds to a line of sight within $10^{\circ}$ of the upwind direction. The dotted line corresponds to a line of sight toward the north ecliptic pole. The vertical dotted lines show the times when SOHO is downwind from the Sun. The polar intensity is very well correlated to the position of SOHO around the Sun, whereas the upwind intensity shows a different and variable behavior.

\subsection{Density Model}

[28] The hydrogen distributions used in this work are derived from the Hot Model [Thomas, 1978; Lallement et al., 1985]. This model assumes that the hydrogen distribution at a large distance from the Sun is unperturbed by the Sun. In that case, the $\mathrm{H}$ atom distribution is given by a Maxwellian distribution characterized by a set of values at infinity, i.e., where the solar influence can be neglected. These values are the number density, the bulk velocity (a vector), and the temperature, respectively noted $N_{H}, V_{H}$, and $T_{H}$. If there is no interaction between the interstellar hydrogen atoms and the heliospheric interface, then those three quantities correspond to the values in the interstellar medium. On the other hand, as shown by Baranov and Malama [1993], the existence of the heliospheric interface and the hydrogen/proton coupling by charge exchange can induce significant changes between the local conditions after crossing the interface region and the actual conditions of the interstellar gas. However, closer to the Sun, both models describe the hydrogen atoms in the same way.

[29] In what follows, we will consider a set of parameters $\left(N_{H}, V_{H}, T_{H}\right)$ at infinity derived from the study of the SWAN hydrogen absorption cell data [Lallement et al., 2005]. The direction of the interstellar wind is also derived from the same data set [Koutroumpa et al., 2005]. The values are given in Table 2. These values characterize the hydrogen distribution at large distance from the Sun but still inside the heliospheric interface, i.e., after modification from interstellar values by the crossing of the interface region. The hydrogen number density value used here is compatible with the estimate given by Gloeckler and Geiss [2004] after the heliopause crossing.

[30] As the hydrogen atoms come closer to the Sun, they are affected by the solar gravitational pull, which is an attractive force but also by the repulsive effect of the radiation pressure. Both forces have the same dependence on distance from the Sun because the solar flux varies as the inverse of the square of the distance to the Sun. The ratio of radiation pressure over solar attraction is noted $\mu$. This parameter changes during the 11-year solar activity cycle with values ranging between 0.9 (attractive case) and 1.6 (repulsive case) [de Toma et al., 1996]. In the present model, we assume that the radiation pressure for the date of observation is the same for all heliographic latitudes.

[31] In the vicinity of the Sun, neutral hydrogen atoms are ionized by charge exchange with the solar wind protons or photoionized by EUV photons. Once again, both ionizing fluxes are proportional to the inverse of the square of the distance to the Sun. Consequently, the total ionization rate $\beta_{\text {tot }}$ is characterized by its value at $1 \mathrm{AU}$. This is the parameter that we wish to determine here. Early works by Kumar and Broadfoot [1979] using Mariner 10 UVS data, or Lallement et al. [1985] using Prognoz 5/6 data, have

Table 2. Insterstellar Parameters for the Hot Model

\begin{tabular}{lc}
\hline \multicolumn{1}{c}{ Parameter } & Value \\
\hline$N_{H}$ & $0.09 \mathrm{~cm}^{-3}$ \\
$V_{H}$ & $22 \mathrm{~km} / \mathrm{s}$ \\
$T_{H}$ & $13,000 \mathrm{~K}$ \\
Upwind Direction (ecliptic J2000) & $252.5^{\circ} \pm 0.50^{\circ}, 8.8^{\circ} \pm 0.50^{\circ}$ \\
\hline
\end{tabular}


demonstrated that the ionization rate varies with heliographic latitude. This has been taken into account in the modeling by discretizing the total ionization rate on a given number of values covering the range of heliographic latitude between $-90^{\circ}$ to $+90^{\circ}$. When computing the extinction suffered by hydrogen atoms on their trajectory through the heliosphere, the heliographic latitude of the subsolar point is computed and the corresponding total ionization rate is used.

[32] In summary, the local hydrogen density distribution is defined by a set of three outer parameters $\left(N_{H}, V_{H}, T_{H}\right)$, combined with the solar parameters $\mu$ and $\beta_{\text {tot }}$. The dynamic parameter $\mu$ characterizes the effect of radiation pressure versus gravitational pull of the Sun on the hydrogen atom trajectories. The total ionization $\beta_{\text {tot }}$ is a represented by a function of heliographic latitude. All variations on heliographic longitude are averaged out because the solar rotation period is short as compared to the hydrogen lifetime against ionization.

\subsection{Line of Sight Integration}

[33] The interplanetary Lyman $\alpha$ background is computed by integration of the spectral volume emission on the line of sight multiplied by extinction between the scattering point and the observer. The spectral volume emission $\varepsilon(\vec{r}, \vec{\Omega}, \lambda) d \lambda$ expresses the number of photons emitted per second in direction $\vec{\Omega}$ at wavelength $\lambda$ per volume unit and per solid angle.

[34] In the optically thin approximation, the total volume emission (emissivity) is given by

$$
\begin{aligned}
\varepsilon_{t o t}(\vec{r}) & =\int_{4 \pi} \int_{0}^{\infty} \varepsilon(\vec{r}, \vec{\Omega}, \lambda) d \lambda d \Omega=g(\vec{r}) N(\vec{r}) \\
& =\sigma_{t o t} F_{s}(\vec{r}) N(\vec{r}) .
\end{aligned}
$$

It measures the number of photons emitted per second per volume. The excitation rate $\left(\mathrm{s}^{-1}\right) g(\vec{r})=\sigma_{t o t} F_{s}(\vec{r})$ expresses the probability of a hydrogen atom at position $\vec{r}$ to scatter a Lyman $\alpha$ photon. $F_{s}(\vec{r})$ is the solar flux at Lyman $\alpha$ in photons $\mathrm{cm}^{-2} \mathrm{~s}^{-1} \AA^{-1}$. The scattering cross section of the transition in $\mathrm{cm}^{2} \AA$ is $\sigma_{\text {tot }}$. See Quémerais and Bertaux [1993] for details.

[35] The intensity measured at wavelength $\lambda$ per wavelength interval for an observer at position $\vec{r}$ for a line of sight with direction vector $\vec{\Omega}$ is expressed by:

$$
I(\vec{r}, \vec{\Omega}, \lambda)=\int_{0}^{\infty} \varepsilon(\vec{r}+s \vec{\Omega},-\vec{\Omega}, \lambda) e^{-\tau_{\lambda}(\vec{r}, \vec{r}+s \vec{\Omega})} d s
$$

The quantity $\tau_{\lambda}\left(\vec{r}_{1}, \vec{r}_{2}\right)$ is the optical thickness at wavelength $\lambda$ between two points and $s$ is the length on the line of sight.

[36] In the optically thin approximation, the total volume emission is equal to the excitation rate of the Lyman $\alpha$ line multiplied by the local density. The spectral dependence of the volume emission is defined by the local density distribution projected on the line of sight. Therefore we can write

$$
\varepsilon(\vec{r}, \vec{\Omega}, \lambda)=\frac{\chi(\varphi)}{4 \pi} g(\vec{r}) N(\vec{r}) a(\lambda)
$$

The function $a(\lambda)$ expresses the spectral dependence of the local distribution projected on the line of sight, i.e., it gives the fraction of atoms of the local distribution that can emit a photon at wavelength $\lambda$ toward the observer. Here, we have $\int a(\lambda) d \lambda=1$. The scattering phase function at Lyman $\alpha$ $\chi(\varphi)$ measures the probability of having an angle $\varphi$ between incident and outgoing propagation directions of the photon. The spectral dependence of the emission profile $a(\lambda)$ is computed from the density model presented above.

[37] It should be noted here that this computation scheme is not a true optically thin approximation because the extinction on the line of sight is included. Following Lallement et al. [1985], we call this the self-absorbed approximation.

\subsection{Radiative Transfer Corrections}

[38] In this study we have added a correction for radiative transfer effects as described by Quémerais [2000]. Various studies of radiative transfer at Lyman $\alpha$ in the interplanetary medium have been published [Keller et al., 1981; Hall et al., 1993; Quémerais and Bertaux, 1993; Quémerais, 2000]. They concluded that radiative transfer effects could not be neglected at 1 AU from the Sun because, even though the inner heliosphere is optically thin at Lyman $\alpha$, a nonnegligible component coming from larger distances from the Sun, where the medium is optically thick, contributes to the total intensity.

[39] Quémerais [2000] computed the total emissivity $\varepsilon_{r t}$ in each point of the heliosphere for various hot model distributions of hydrogen, using different sets of parameters $\left(N_{H}, V_{H}, T_{H}\right)$ and $\left(\mu, \beta_{\text {tot }}\right)$. In these computations, the ionization rate was chosen to be isotropic with heliographic latitude. By comparison with optically thin emissivity values $\varepsilon_{o t}$ computed for the same density model (equation (3)), it is possible to define a correction ratio $R_{c o r}$ that expresses the difference between full radiative transfer emissivity and optically thin emissivity.

$$
R_{c o r}(\vec{r})=\frac{\varepsilon_{r t}(\vec{r})}{\varepsilon_{o t}(\vec{r})}
$$

It was found that this correction ratio is mostly affected by the value of $N_{H}$ outside $10 \mathrm{AU}$ from the Sun where solar parameters have less effect on the distribution of hydrogen atoms.

[40] So to correct for radiative transfer effects in our computation, we multiply the emissivity from equation (3) by the correction factor described above. This correction factor has been initially computed for the same values of interstellar parameters and average values of $\left(\mu, \beta_{t o t}\right)$. The correction is applied during the integration on the line of sight. This correction has no wavelength dependence.

[41] Figure 5 shows the typical correction applied to the models. This figure shows the ratio of the intensity corrected for multiple scattering divided by the uncorrected intensity. Multiple scattering corrections are more important for lines of sight close to the downwind direction [Quémerais, 2000].

[42] Including this correction has strongly improved the overall fit of the SWAN data. Its main effect is to increase the total intensity measured in the downwind cavity by a factor of about 1.6 relative to the upwind intensity. Upwind intensities are not much affected by this correction. Models using this correction yield upwind to downwind intensity 

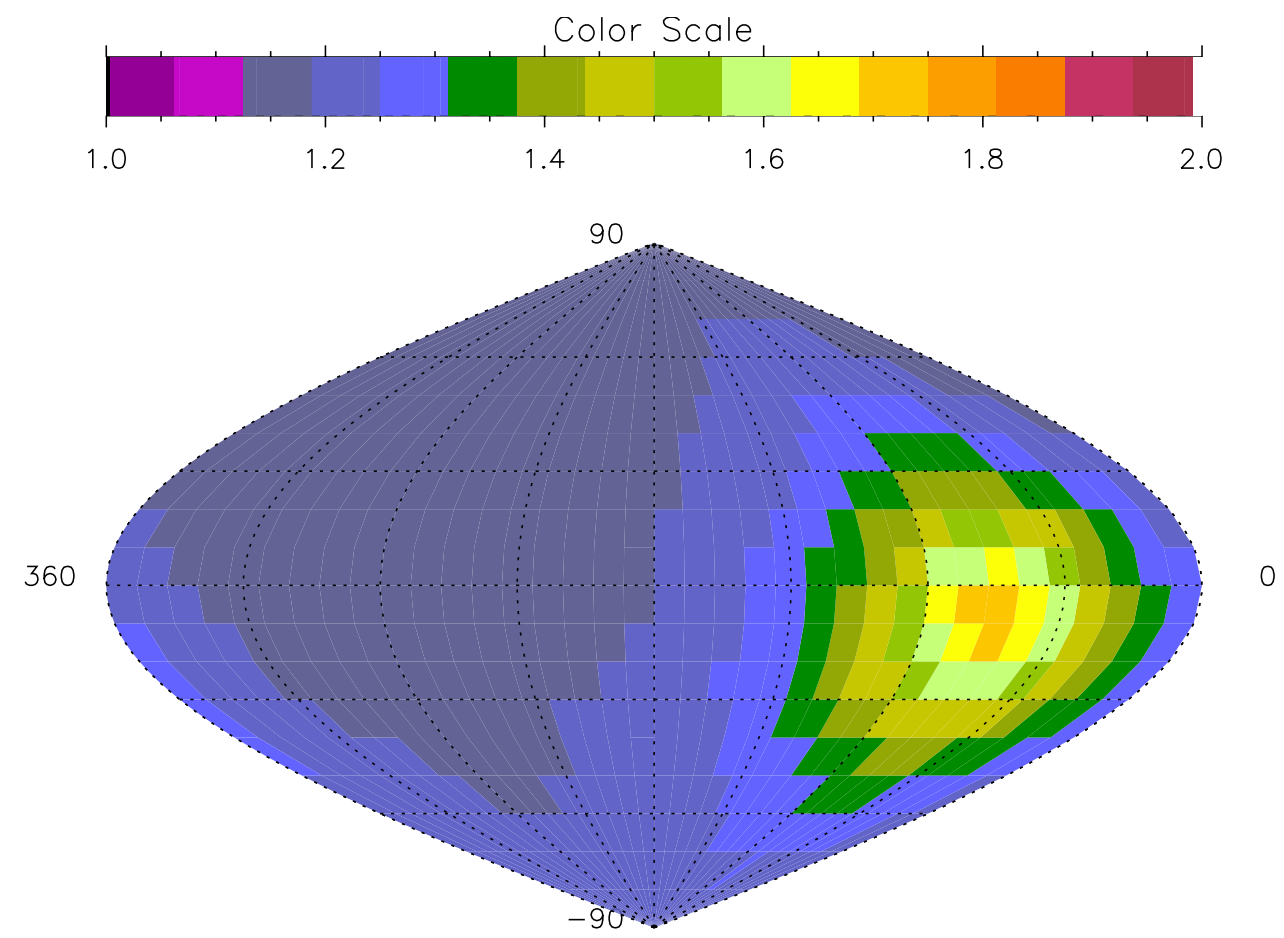

Figure 5. Example of radiative transfer correction computed for a full sky map observed by SWAN in September 1996. The figure shows in ecliptic coordinates the ratio of the model intensities divided by a fully optically thin model (i.e., there is no autoabsorption on the line of sight). The radiative transfer intensity is larger by about $10 \%$ in the upwind direction. In the downwind direction the ratio is close to 1.7. The upwind to downwind intensity ratio is strongly affected by radiative transfer corrections.

ratios that are much closer to observed values than models without such correction.

\subsection{Solar Flux and Radiation Pressure}

[43] During the 11-year solar cycle, the solar Lyman $\alpha$ illuminating flux varies by a factor close to 1.8 [de Toma et al., 1997]. This value is based on the composite data set produced by Woods et al. [2000], which includes measurements from various space experiments.

[44] We have used this data set to produce the illuminating flux at the time of observation $F_{S}$ and the radiation pressure coefficient $\mu$ necessary in the model computation. However, the data set published by Woods et al. [2000] concerns the total solar Lyman $\alpha$ flux. In the case of interplanetary hydrogen atoms, only the line core flux is relevant because of the small Doppler shift between the hydrogen atoms and the Sun. Looking at the solar Lyman $\alpha$ spectra published by Lemaire et al. [2002] from measurements made by SUMER/SOHO, we found that the ratio of the core flux to the total flux is equal to $0.9 \AA^{-1}$. Consequently, all fluxes values are multiplied by 0.9 to account for this ratio between the line core flux and the total flux.

[45] The data used in the fitting procedure are averaged over 1 month of observations to smooth out anisotropies of the solar illuminating flux [Bertaux et al., 2000]. Consequently, the flux value used in the model computation is an average of the solar flux measured on the dates of the observations. This tends to smooth out 27-day rotational variations of the solar illuminating flux.
[46] The radiation pressure coefficient was derived from the same data set. Here $\mu$ is the ratio of radiation pressure modulus over gravitational attraction modulus. A value of $\mu$ larger than 1 means that the residual force felt by hydrogen atoms is repulsive. Here again a factor 0.9 was used to account for the change from total flux measurement to line core flux value. We found that $\mu$ varies between 0.9 during solar minimum and reaches 1.6 at solar maximum or a few months later. For each map we have averaged $\mu$ over the 6 months preceding the observation. Averaging smoothes out rapid variations of the Lyman $\alpha$ flux on timescales which are shorter than the typical travel time of the hydrogen atom in the inner heliosphere.

[47] Figure 6 shows the values which have been used as parameters to fit the monthly averaged data. The values range between 0.9 and 1.7. The squares show the mean illuminating solar flux used for the month of data, normalized to $3.32 \times 10^{15} \mathrm{~m}^{-2} \mathrm{~s}^{-1} \AA^{-1}$. The radiation pressure coefficient values are shown by the diamonds. This second parameter is smoother because it is an average of 6 months of data before the time of observation. Except for a few months in 1997, the radiation coefficient $\mu$ is larger than 1 most of the time. This means that the net force felt by the hydrogen atoms in the inner heliosphere is repulsive. Earlier analyses from the 1980s [e.g., Kumar and Broadfoot, 1979; Bertaux et al., 1985] often used values of $\mu \leq 1$. Values of the radiation coefficient smaller than 1 were deduced from available measurements of the solar H Lyman $\alpha$ illuminating flux. These values were revised upward after the 


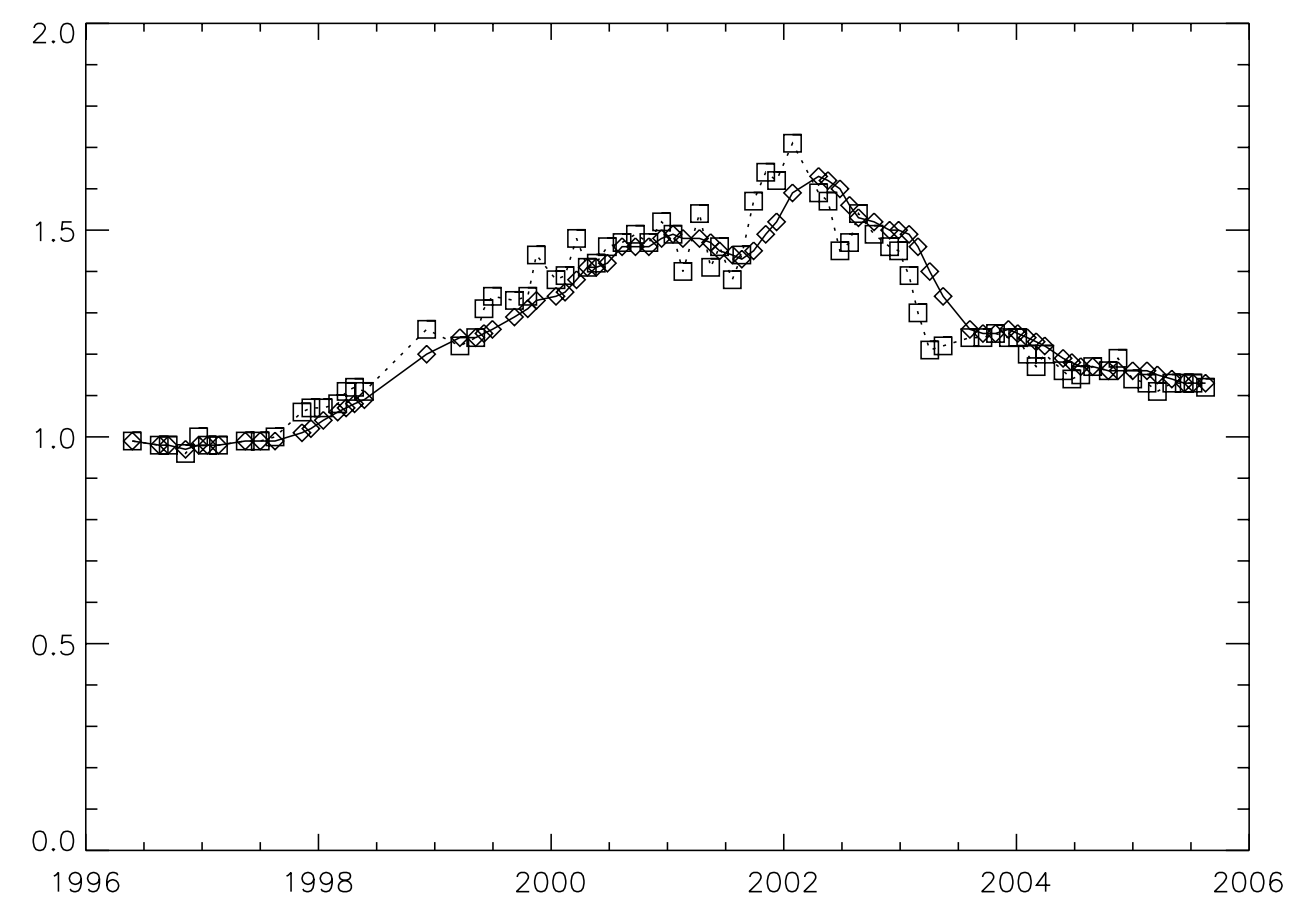

Figure 6. Solar flux values and radiation pressure coefficient used to fit the data. These values are parameters in the fitting procedure. They are derived from the data set of solar Lyman $\alpha$ flux published by Woods et al. [2000]. The solar Lyman $\alpha$ flux values are normalized to $3.32 \times 10^{11}$ photons $\mathrm{cm}^{-2} \mathrm{~s}^{-1}$ $\AA^{-1}$.

measurements of the SOLSTICE/UARS were obtained. Many of the previous analyses should be revised to account for the correct radiation pressure coefficient.

\section{Results: Total Ionization}

[48] Figure 7 shows how the intensity varies during the solar cycle once the solar illuminating flux variations have been removed. For this plot, we have used only two lines of sight which give a good idea of the general pattern. The original data are shown in Figure 4. After correction for the variations of the illuminating flux, we clearly see that the hydrogen cavity around the Sun increases with the solar cycle. It is partly due to the increase of radiation pressure that deflects hydrogen atoms away from the Sun. Also, ionization is expected to become more efficient with increasing activity.

[49] The total ionization rate at $1 \mathrm{AU}$ for hydrogen is derived by fitting the monthly averaged data maps (i.e., between 1500 and 2000 individual lines of sight) with the model described above. All the parameter models are fixed except for the 19 values of the ionization rate as a function of heliographic latitude. The fitting algorithm is based on the Levenberg-Marquardt algorithm described in Numerical Recipes [Press et al., 1986].

\subsection{Absolute Value}

[50] Table 2 shows the values of the interstellar parameters used in this analysis. The fit has been performed on 75 monthly averaged maps, covering a 10 -year period. Each fit requires about 5 or 6 iterations before convergence is achieved.
[51] Figure 8 shows the resulting hydrogen ionization rate as a function of time. The quantity displayed is the value averaged over the whole Sun, such as

$$
\langle\beta\rangle=\int_{-\pi / 2}^{\pi / 2} \beta(\lambda) \frac{\cos (\lambda)}{2} d \lambda,
$$

Here, $\lambda$ is the heliographic latitude.

[52] This quantity gives a global view of how the ionization rate changes with solar activity. It is also a good indicator of the size of the ionization cavity. We see that the total ionization rate at $1 \mathrm{AU}$ varies roughly by a factor of 2 during the solar cycle. What is more striking is that although ionization roughly follows solar activity, the maximum of the ionization rate happens later (2003-2004) than the sunspot number maximum (2001). This is due to the ionization rate's being the sum of two ionization processes, photoionization, which has its maximum in 2001 and ionization by charge exchange with solar protons. This second hydrogen ionization process seems to be maximum at a later period than the sunspot number maximum.

[53] The values shown in Figure 8 display an annual modulation between 2001 and 2005. In the period from 1996 to 2001, this modulation does not show clearly. The amplitude of the modulation represents about $10 \%$ to $15 \%$ of the total ionization rate. The maximum values are found in December when $\mathrm{SOHO}$ is in the downwind direction from the Sun. The correlation between the position of the spacecraft around the Sun and the modulation of the ionization rate suggests that this is an artefact of the analysis. 


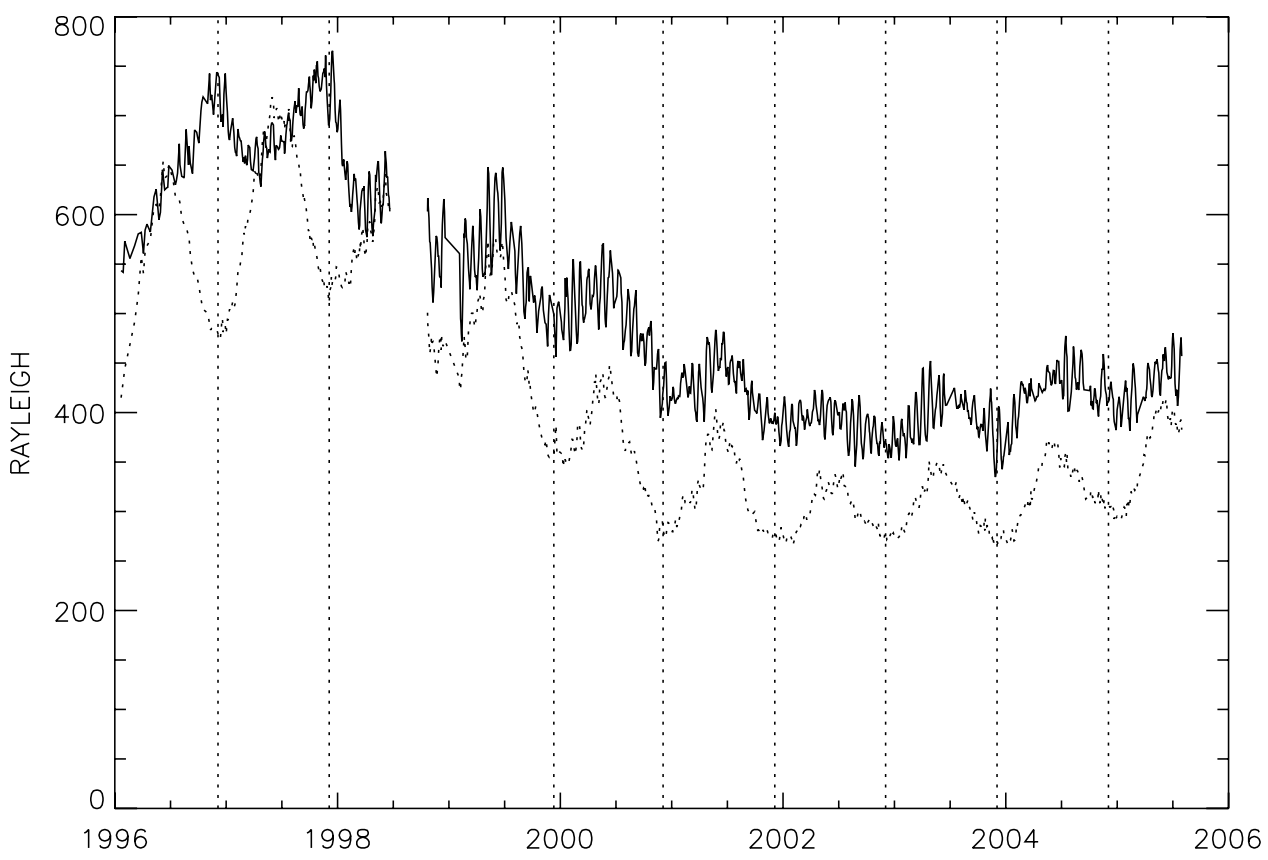

Figure 7. IP background intensities for two lines of sight as a function of time. The two lines of sight are close to the upwind direction (solid line) and north ecliptic direction (dotted line). The data are the same as the ones shown in Figure 4 . Here the values are divided by $\mu$ to correct for the variation of the solar illuminating flux. We clearly see that the IP hydrogen density decreases during the solar cycle due to the change in radiation pressure and solar ionization.

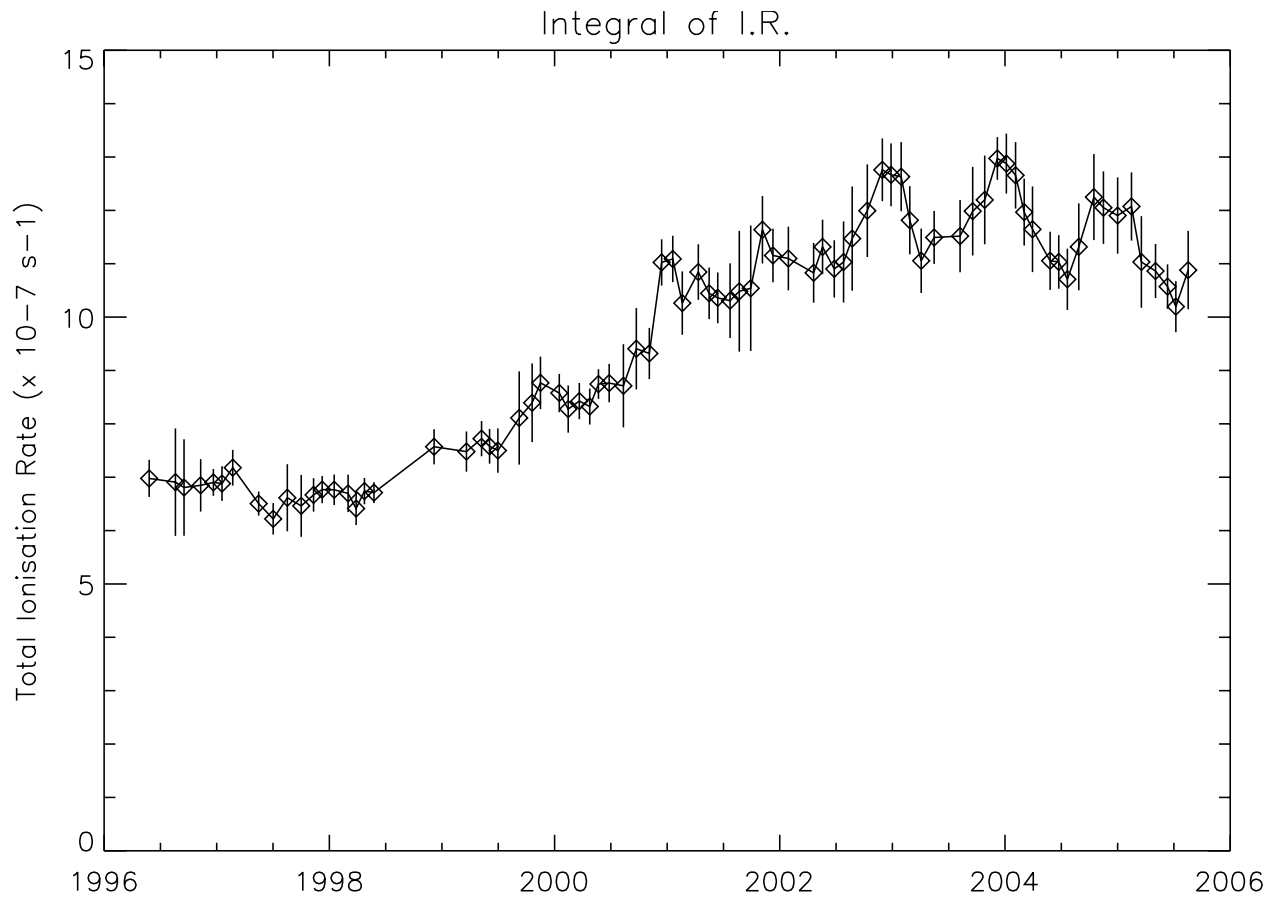

Figure 8. The plot shows the solar cycle variations of the total ionization rate of hydrogen at 1 AU, averaged over the whole Sun. The variation between minimum and maximum is roughly a factor of 2 . The peak value is reached almost 2 years after the sunspot number maximum in 2001. The annual modulation which appears after 2001 is an artefact of the analysis. 


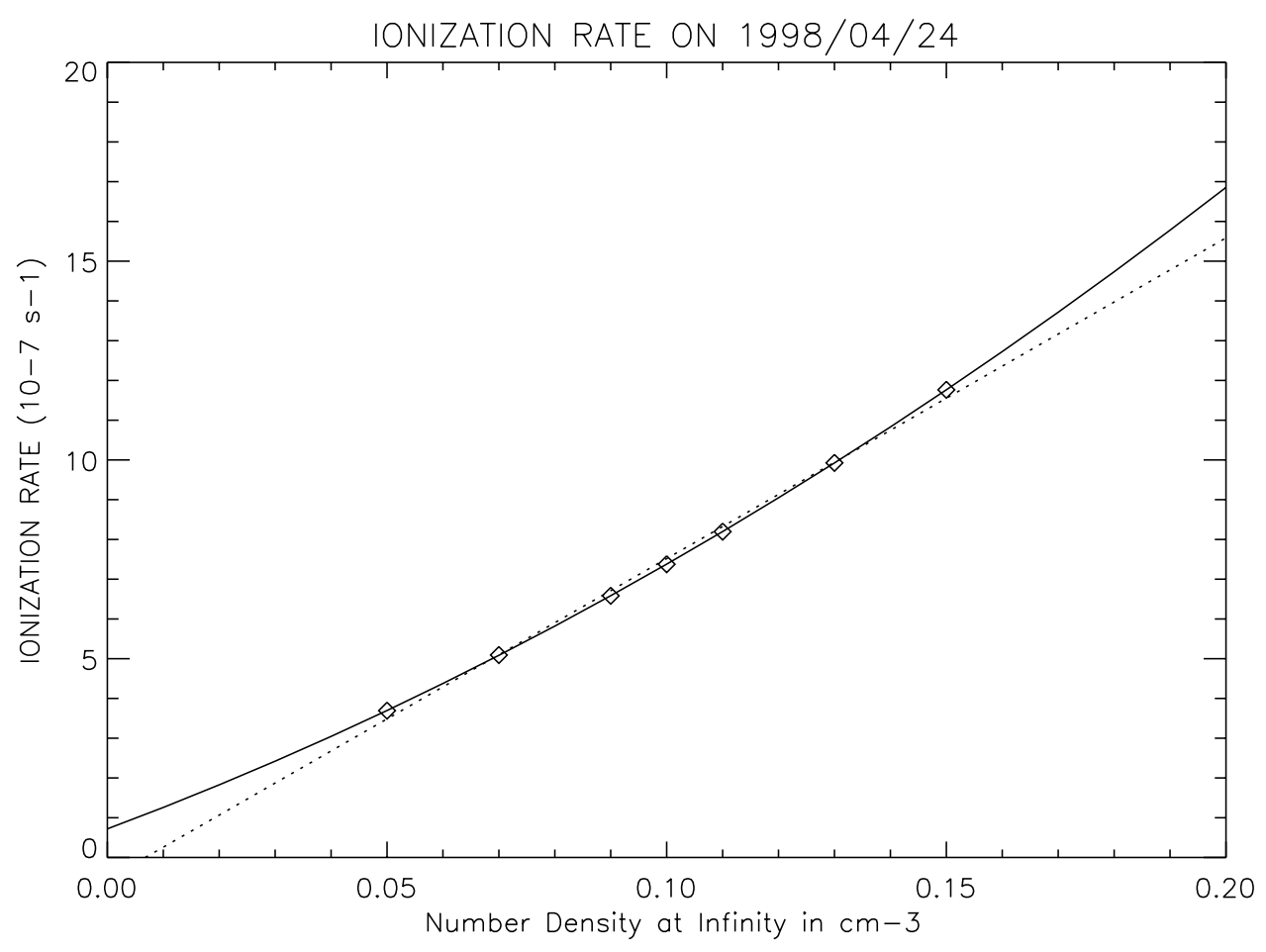

Figure 9. Total ionization rate at $1 \mathrm{AU}$ as a function of the Hydrogen number density at infinity. This plot shows how the absolute value of the ionization rate depends on the assumed value of the hydrogen number density. Usual values of the hydrogen number density range between 0.05 and $0.15 \mathrm{~cm}^{-3}$. The values were computed for a SWAN map in April 1998. We find a near linear dependence between the total ionization rate and the hydrogen number density (dotted line). The best fit is obtained by a second order polynomial relation (solid line).

\subsection{Number Density Parameter}

[54] One of the strongest assumptions made in this analysis corresponds to the value of $N_{H}$ which is a scaling factor for the hot model. The chosen value $N_{H}=0.09 \mathrm{~cm}^{-3}$ is consistent with recent analyses. However, it is significantly smaller than the value of $0.13-0.17 \mathrm{~cm}^{-3}$ which was obtained by previous analyses [Quémerais et al., 1994; Pryor et al., 1996].

[55] Therefore we have chosen to evaluate the impact of the value of this parameter on the ionization rate derived in the present work. We have selected one of the maps (April 1998) and applied the fitting procedure to the data with various values for $N_{H}$ ranging between $0.05 \mathrm{~cm}^{-3}$ and $0.15 \mathrm{~cm}^{-3}$. For each number density at infinity, the radiative transfer correction has been computed as described above and applied in the analysis.

[56] Figure 9 shows the Sun-averaged ionization rate $\langle\beta\rangle$ as a function of $N_{H}$, the hydrogen number density at infinity. The relation found here is quasi-linear, suggesting that if the density is different for $0.09 \mathrm{~cm}^{-3}$ in the outer heliosphere, a simple linear correction can be applied.

[57] However, it must be noted that all the fits are far from being equivalent. The best data-to-model fit is obtained for $N_{H}=0.09 \mathrm{~cm}^{-3}$. Models using values of $N_{H}=0.05 \mathrm{~cm}^{-3}$ and $N_{H}=0.15 \mathrm{~cm}^{-3}$ give a very bad fit to the data because, for such densities, the model can not represent correctly the observed ratio between upwind and downwind intensities. This exercise also shows that the SWAN data are best represented by a hot model using an hydrogen number density at infinity equal to $0.09 \mathrm{~cm}^{-3}$.

\subsection{Anisotropy of the Ionization Rate}

[58] The primary science objective of the SWAN instrument is to characterize the anisotropy of the solar wind mass flux. To do that, we need to quantify the anisotropy of the hydrogen ionization rate. This is done in Figures 10 and 11.

[59] The first one (Figure 10) shows the latitudinal and temporal variations of the total hydrogen ionization rate. The color scale gives the value of the ionization rate as a function of time ( $\mathrm{x}$ axis) and heliographic latitude (y axis). The values are sampled every 10 degrees between $-20^{\circ}$ and $+20^{\circ}$ and every 20 degrees for higher latitudes. This plot shows the global increase of the ionization rate from 1996 to 2001. After 2000, high values of ionization rates can be found at all latitudes.

[60] The second one (Figure 11) shows the same data divided by the mean equatorial value (average from $-20^{\circ}$ to $+20^{\circ}$ ). We see the shift from an anisotropic ionization rate pattern at solar minimum to an isotropic pattern at solar maximum. The period when the ionization rate is actually isotropic is rather small, in 2001. Soon after 2001, ionization rates at the poles start to decrease. There is also a small systematic north-south asymmetry in 2002 and 2003. Hydrogen ionization rate anisotropies can also be represented by the ratio of the equator value over pole values.

[61] Figure 12 shows the polar and equatorial values of the total ionization rate as a function of time in solar 

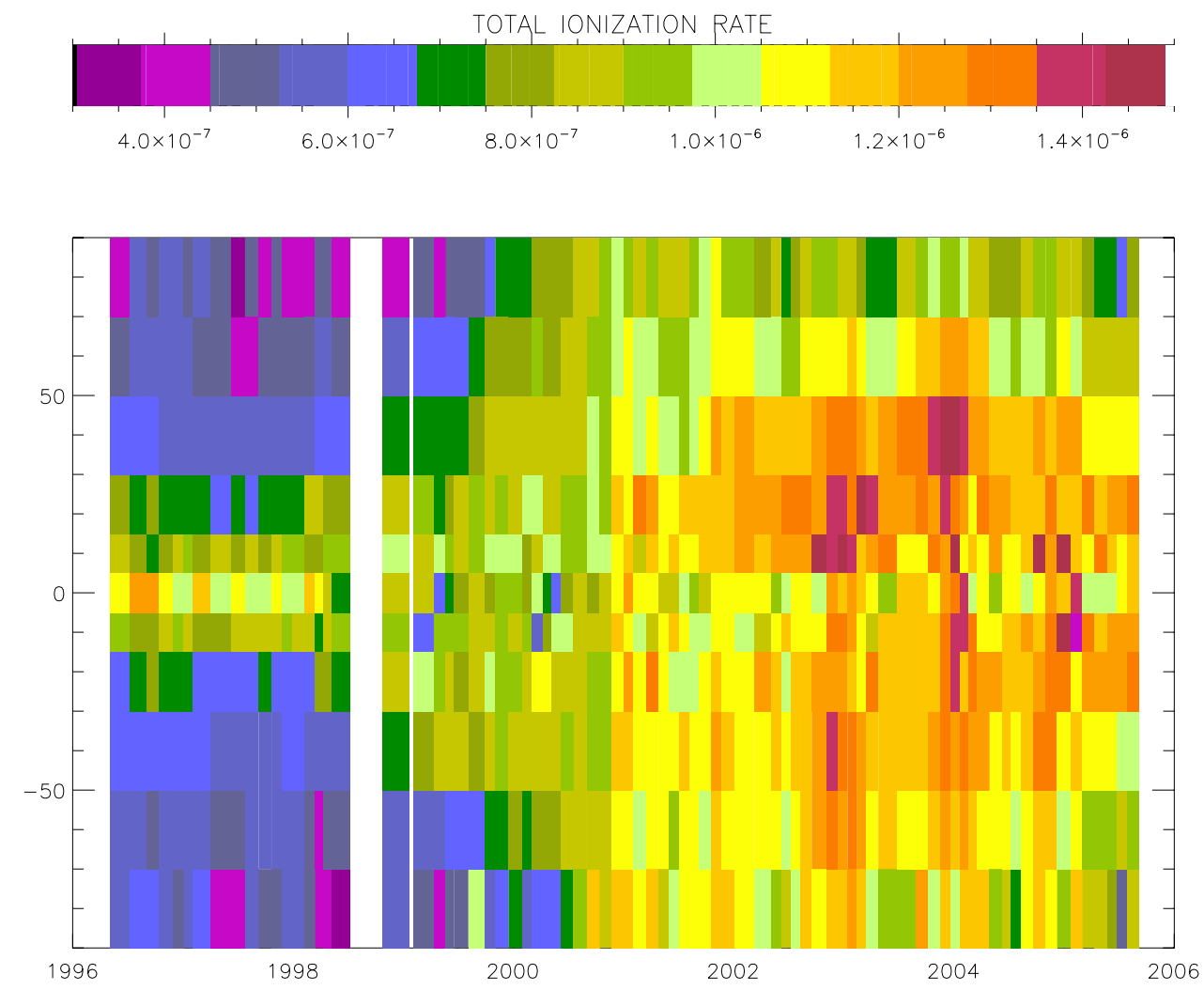

Figure 10. Latitudinal and temporal distribution of the total ionization rate. The $\mathrm{x}$ axis is the time of observation, the $\mathrm{y}$ axis is the heliographic latitude. The color scale of the $\mathrm{z}$ axis (ionization rate) is shown at the top. There are two data gaps in 1998 and early 1999. The enhanced ionization along the solar equator is clearly visible from 1996 to 1999. After 2000, high values of ionization rates can be found at all latitudes.

cycle 23. The equatorial values are averaged between latitudes -20 to +20 . The polar values are averaged for heliographic latitudes larger than +40 (north pole) or smaller than -40 (south pole). Within statistical errors of our estimates, the equatorial value is always larger than the polar values. At best during solar maximum, the polar values are very close to the equatorial values but never significantly larger. In the period of 2001 to 2002, the south polar ionization rate is systematically larger than the north polar ionization rate. This is not the case for the rest of the cycle 23 shown in this figure.

[62] Figure 13 shows the ratio of the equatorial value of the ionization rate divided by the average of the polar values. The ionization is strongly asymmetric between 1996 and 2000 with an excess of $70 \%$ in 1998. This was shown early on during the SOHO mission [Bertaux et al., 1997; Kyrölä et al., 1998]. It corresponds to the period when the slow solar wind is concentrated along the solar equator causing a much larger ionization by charge exchange that of the fast solar wind, which has a significantly reduced mass flux.

[63] The ionization rate is roughly symmetric between the second half of 2000 and 2004, although even in this period the equatorial ionization rate is still slightly larger than the pole values. During this period of the cycle, fast and slow solar winds are mixed at all heliographic latitudes. The resulting ionization rate by charge exchange with the solar wind is therefore more or less constant with heliographic latitude.

[64] After 2004, the anisotropy of the ionization rate reappears clearly and is increasing rapidly. At the end of 2005 , the ratio is already close to 1.4 . We expect that this ratio will keep on rising until the next solar minimum in 2006 or 2007 . Whether the anisotropy will be the same in 2006 and 2007 as the one observed in 1996 is a matter for future observations by the SWAN instrument.

\section{Solar Wind Flux Values}

[65] The present analysis shows how the total ionization rate for hydrogen in the interplanetary medium and its latitudinal variations can be deduced from the SWAN data. The total ionization rate of hydrogen is generally considered to be the sum of two terms. The first one, $\beta_{E U V}$, is due to photoionization by EUV photons from the Sun. The second one, $\beta_{e x}$, is caused by charge exchange with the protons of the solar wind.

[66] There is a third process that can contribute to the ionization of hydrogen in the vicinity of the Sun. Electron impact ionization is usually considered to be negligible outside the orbit of the Earth because the cross section of the ionization is very dependent on electron temperature. However, some works on He have shown that it may be more effective than what is usually assumed [Lallement et 

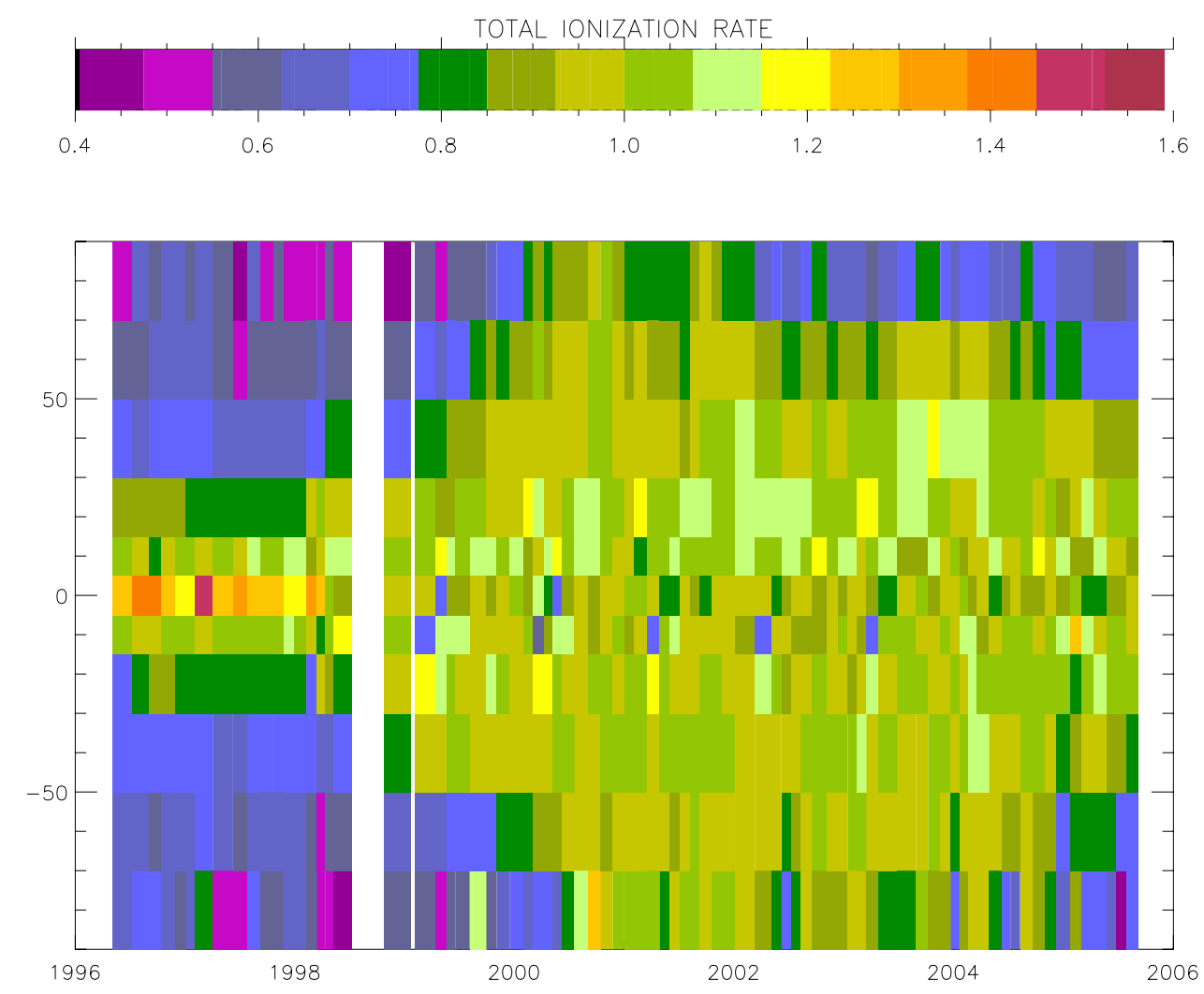

Figure 11. Latitudinal and temporal variations of the relative ionization rate. For each month, the values shown in the previous graph are normalized to the average value between heliographic latitudes of $-20^{\circ}$ to $20^{\circ}$ (equatorial values). This plot shows how the pattern evolves during the solar cycle, from a strong equatorial enhancement at solar minimum to an isotropic distribution in 2001. After 2001, ionization quickly decreases at the poles.

al., 2004], and we may need to include it in future analyses. For now, we will neglect it here.

\subsection{Deriving Solar Wind the Mass Flux From SWAN Data}

[67] To derive the absolute value of $\beta_{e x}$, which is linked to the solar wind mass flux, it is necessary to know the absolute value and spatial and temporal variations of $\beta_{E U V}$. A full analysis of the hydrogen photoionization term is outside the scope of this work. However, using previous estimates, we can see how this affects our results. Numerical estimates of the ecliptic ionization rate are given by Ajello et al. [1987] or Ogawa et al. [1995]. During solar minimum conditions (1977), Ajello et al. [1987] have estimated the photoionization rate to be $0.55 \times 10^{-7} \mathrm{~s}^{-1}$. For solar maximum conditions, they found a photoionization rate at 1 AU equal to $1.2 \times 10^{-7} \mathrm{~s}^{-1}$. Ogawa et al. [1995] give larger values for the photoionization rate. The solar minimum rate is equal to $1.5 \times 10^{-7} \mathrm{~s}^{-1}$ and the solar maximum rate is found to be $2.4 \times 10^{-7} \mathrm{~s}^{-1}$. These values are estimated from in-ecliptic EUV flux measurements. Here, we will assume that the photoionization rate is isotropic. A more detailed study in a future work will have to address and quantify this question.

[68] The total ionization rate derived in our analysis at solar minimum is equal to $8 \times 10^{-7} \mathrm{~s}^{-1}$ in the ecliptic and $5 \times 10^{-7} \mathrm{~s}^{-1}$ at the poles (Figure 12). Solar maximum values are close to $12 \times 10^{-7} \mathrm{~s}^{-1}$. Using the values for photoionization quoted above, we can deduce the chargeexchange ionization rate. Ajello et al. [1987] also give an estimate for the charge-exchange cross section in the $1 \mathrm{keV}$ energy range equal to $1.7 \pm 0.02 \times 10^{-15} \mathrm{~cm}^{2}$. This can be used to derive a solar wind mass flux at $1 \mathrm{AU}$.

[69] Numerical values are given in Table 3. Case 1 refers to values using the photoionization rates given by Ajello et al. [1987] and Case 2 refers to the values given by Ogawa et al. [1995]. This table shows example of solar wind mass fluxes at the poles and the equator deduced from the numerical values presented above. We find that the mass flux is the same at the poles and the equator at solar maximum, but at solar minimum we find a ratio of the polar value divided by the equatorial value equal to $0.53-0.6$, depending on the actual photoionization rate estimate used.

[70] Although the photoionization rate is always smaller than the charge-exchange rate, we see that at solar minimum and at the poles, the value for the photoionization rate given by Ogawa et al. [1995] represents 30\% of the total ionization rate of the interplanetary hydrogen. A more detailed analysis is required here to accurately remove the photoionization component from the total ionization rate. This should also include any heliographic dependence of the photoionization rate and its potential temporal variations during the solar cycle. 


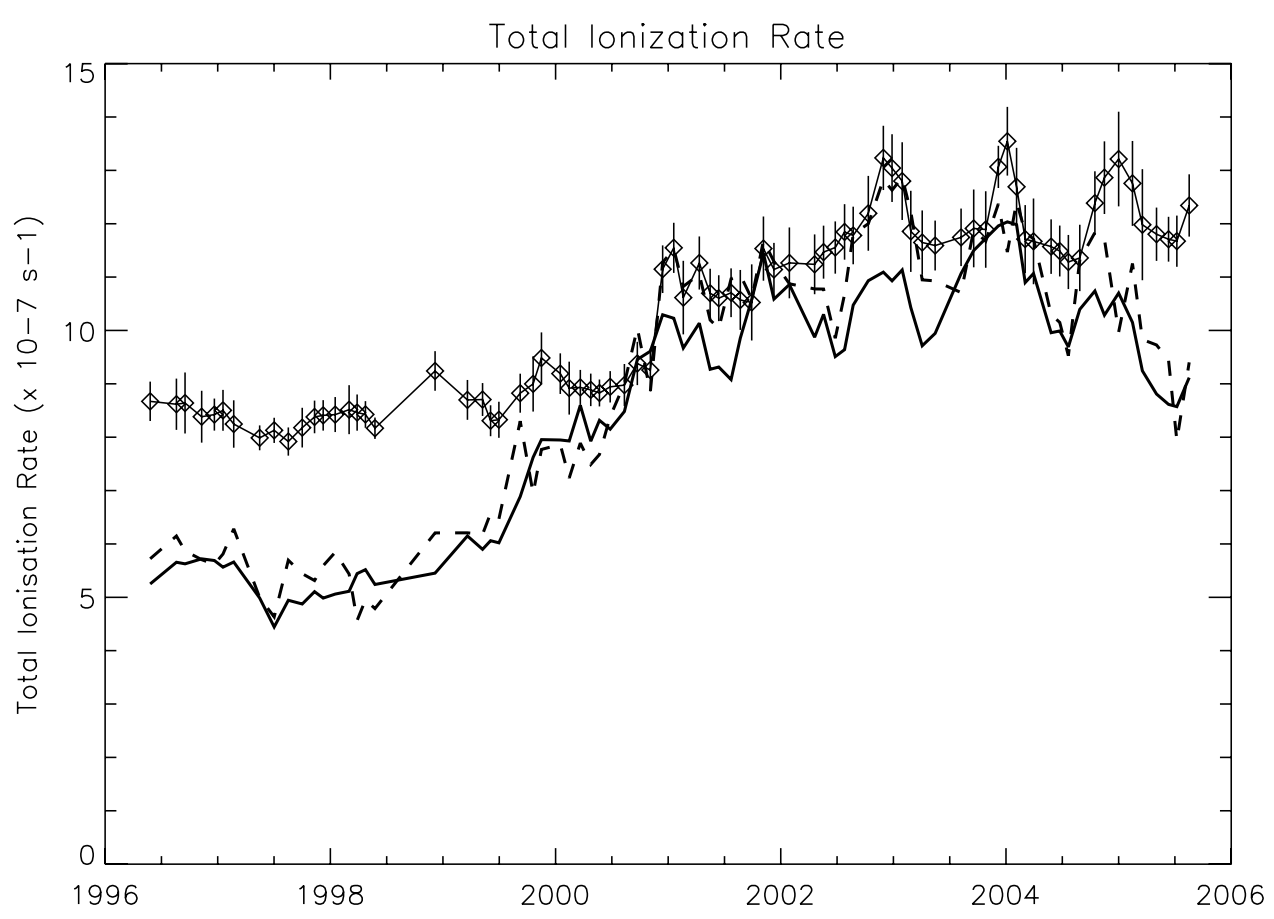

Figure 12. Polar and equatorial values of the total ionization rate as a function of time in solar cycle 23 . The diamonds show the equatorial values, averaged between latitudes $-20^{\circ}$ to $+20^{\circ}$. Equatorial values are larger than the polar values most of the time. The solid line shows the north polar ionization rate averaged for heliographic latitudes above $40^{\circ}$. The dashed line shows the south polar value averaged over latitudes below $-40^{\circ}$. Most of the time, north and south values are very similar, except for a short period in 2001 when the south ionization rate is slightly larger than the north ionization rate. Polar values show a 11-year cycle variation amplitude close to a factor 2 , whereas the equatorial value changes only a factor 1.5 .

\subsection{Comparison With In Situ Measurements}

[71] It is possible to use a different approach to this problem. Various data sets produce daily averages of the solar wind proton velocities and densities as seen from 1 AU. However, these values reflect local conditions in the solar wind, whereas SWAN data reflect global conditions, it is possible to make a comparison to see if the values are compatible.

[72] We have used the data from three instruments covering the period of activity of SWAN. The data used here come from the WIND-SWE instrument, the IMP-8 mission, and the ACE mission. Data were obtained through the public access $\mathrm{ftp}$ sites of the respective instruments. To compute the charge exchange ionization rate from these data, we have used the cross section given by Izmodenov et al. [2001]. The photoionization rate was computed from the values given by Ogawa et al. [1995]. We took the solar minimum and solar maximum values given by these authors and assumed a linear correlation with the solar Lyman $\alpha$ flux.

[73] The sum of these two estimates is shown in Figure 14. The equatorial values found from the SWAN data are also shown for easy comparison. We find a good agreement between the SWAN values and the in situ estimates during solar minimum. However, after 1999, we see an increasing discrepancy between the two methods. As expected from Figure 7, the hydrogen density strongly decreases in the inner heliosphere at solar maximum. Yet according to in situ measurements, the total ionization rate due to photoionization and charge exchange does not increase with activity. Either radiation pressure is solely responsible for the larger size of the hydrogen cavity or another effect is involved here. This is discussed in the next section.

\section{Conclusion and Future Works}

[74] In this work, we have shown how the SWAN interplanetary (IP) Lyman $\alpha$ measurements can be used to estimate the total ionization rate of hydrogen in the interplanetary medium. In principle, these results allow us to derive solar wind mass fluxes and their variations during the solar activity cycle.

[75] Thanks to a new estimate of the absolute calibration of the SWAN instrument and its variation during the 10 years of operation of SOHO, we are able to derive absolute values of the ionization rate as well as its latitudinal dependence.

[76] We have shown how the anisotropy of the ionization rate changes from solar minimum to solar maximum. At solar maximum, the so-called ionization groove [Bertaux et al., 1995] has completely disappeared, and the ionizing fluxes are the same at all heliographic latitudes. Similar results were found by Ulysses SWOOPS team using the proton velocity and density data to estimate the hydrogen ionization rate from charge exchange [McComas et al., 1999; Pryor et al., 2003]. The solar minimum flyby data 


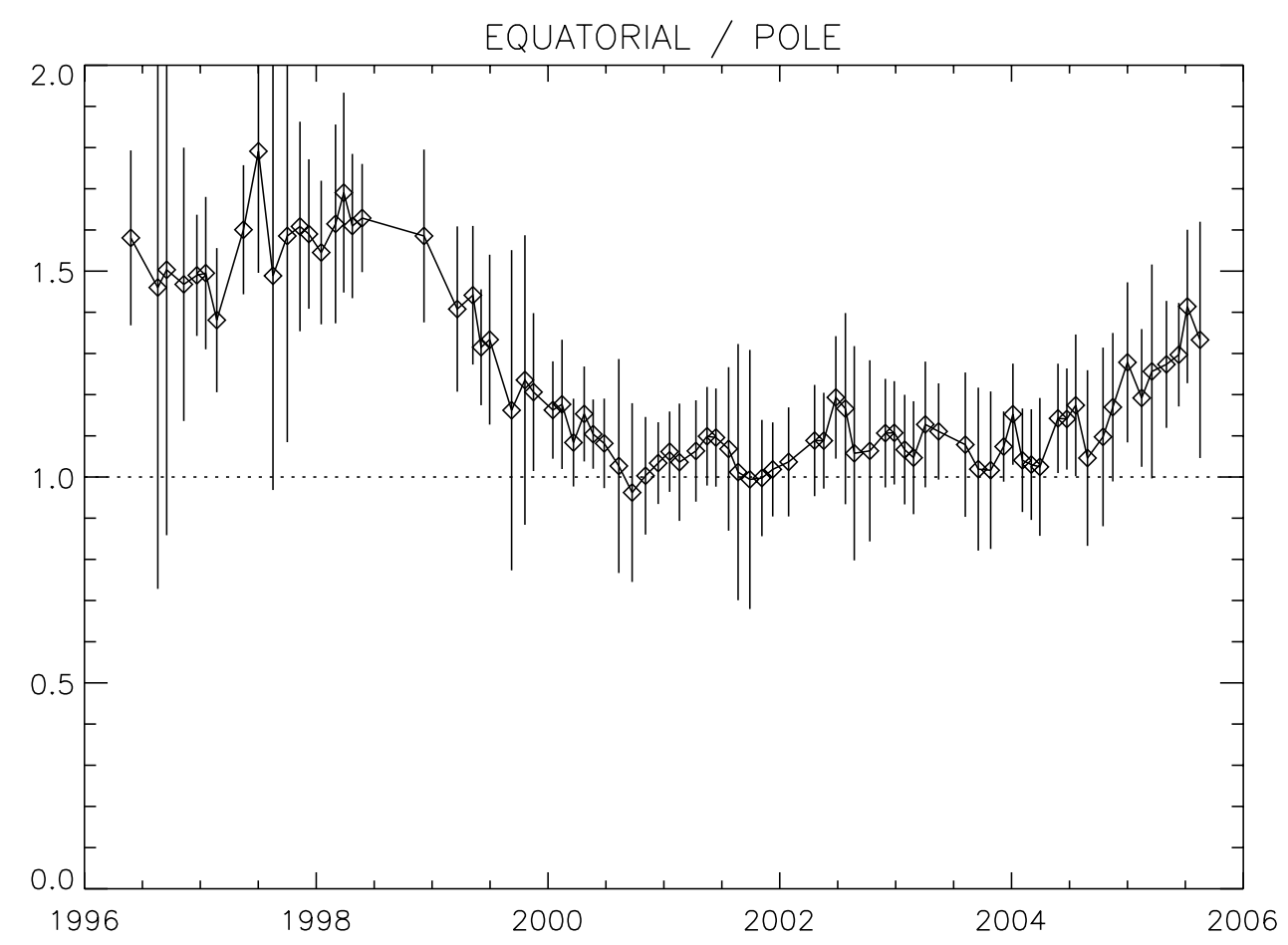

Figure 13. Ratio of the equatorial value of the ionization rate divided by the average of the polar values. This figure shows the amplitude of the asymmetry of the ionization rate and its variation with the solar cycle. The ionization is strongly asymmetric between 1996 and 2000 with an excess of $70 \%$ in 1998 . The ionization rate is roughly symmetric between the second half of 2000 and 2004, although even in this period the equatorial ionization rate is still slightly larger than the pole values. After 2004 the anisotropy of the ionization rate reappears clearly and is increasing rapidly. At the end of 2005, the ratio is already close to 1.4 .

obtained in 1995 show a variation of the hydrogen ionization rate by a factor of 2 from the poles to the equator. This is close to what we find in Table 3. Data from the solar maximum flyby show a quasi-constant ionization rate with heliocentric latitude as shown in Figure 11 and Table 3.

[77] We have found that the hydrogen ionization cavity which surrounds the Sun increases in size with solar activity. This is evidenced by the low interplanetary Lyman $\alpha$ data measured during and after the solar maximum. Our model calculations also show that the increased radiation pressure is not sufficient to explain the larger cavity observed at solar maximum. Indeed, the change in $\mu$ values is taken into account when we perform the fits.

[78] We have shown also that ionization rates derived from in situ solar wind measurements do agree with the SWAN results at solar minimum but are significantly smaller at solar maximum. In situ derived ionization rates do not show the solar cycle dependence we find from the SWAN data.

[79] Since radiation pressure alone cannot explain the change in the Lyman $\alpha$ background pattern, there are a few other possibilities that we will discuss here. First, the estimate of the radiation pressure is based on measurements of the total solar intensity at Lyman $\alpha$ and some measurements of the solar Lyman $\alpha$ line profile. We do not believe that the solar flux estimate is wrong but we must at least consider this. However, to explain the large cavity at solar maximum, we would need values of $\mu$ larger than 2 which does not seem possible.

[80] Second, electron impact ionization on helium has been shown to be more efficient than that is usually assumed [Lallement et al., 2004]. It is possible that a similar effect is important for hydrogen. However, we find that the ionization rates derived at solar minimum by both methods are compatible. This means that there is a strong variation in the efficiency of electron impact ionization with solar activity. To check this, we will need to compute estimates

Table 3. Solar Wind Mass Flux Values at the Poles and the Equator

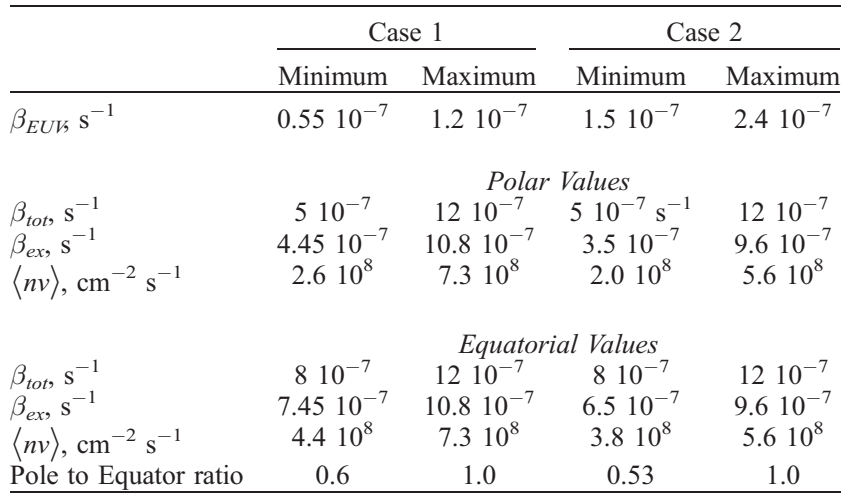




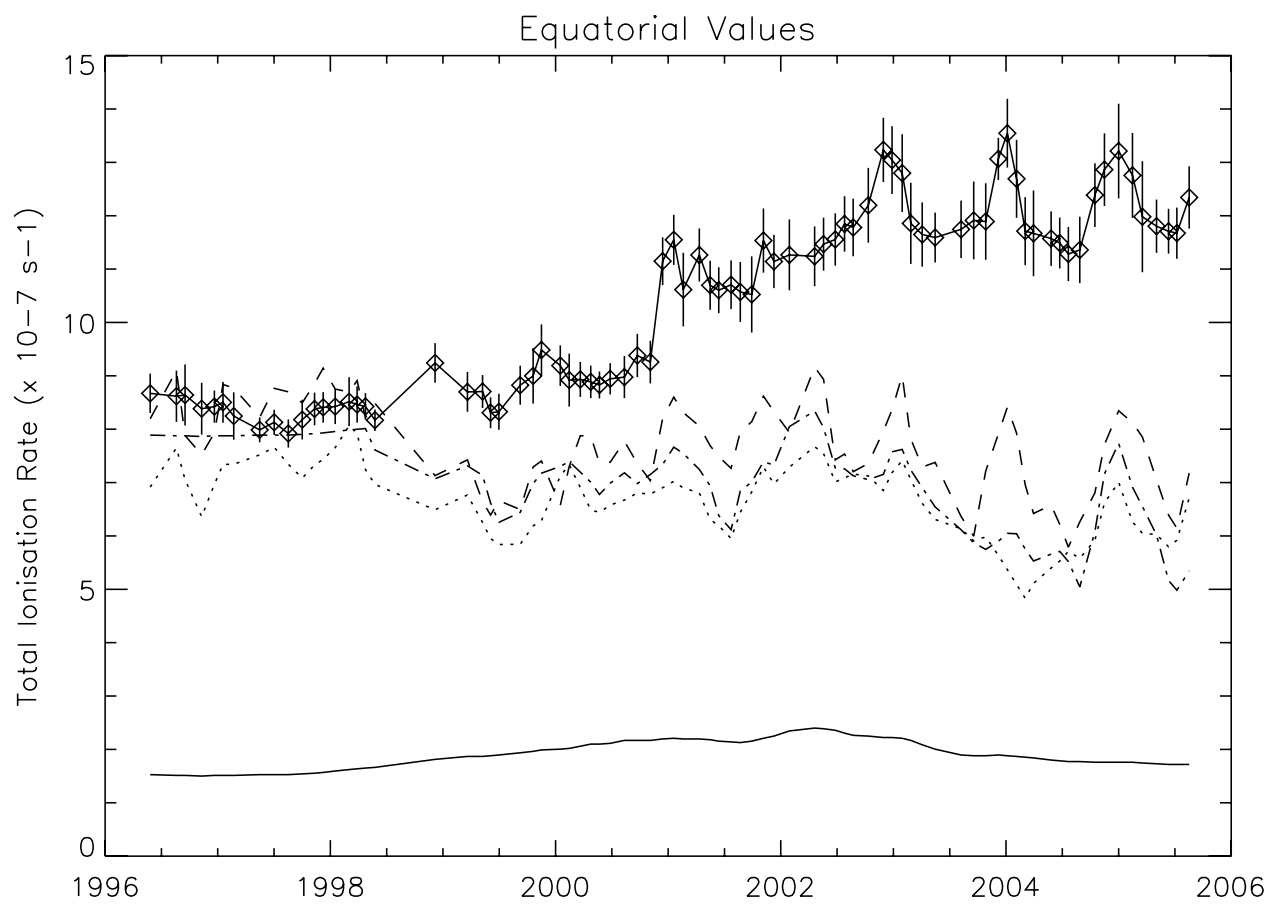

Figure 14. Comparison of equatorial ionization rate values derived in this analysis with ionization rates computed from in situ solar wind measurements (Wind, ACE, and IMP-8 data sets). The photoionization rate is shown by the bottom solid line and is derived from estimates by Ogawa et al. [1995]. The three curves (dashed line, dotted line, dash-dot line) give the sum of the estimates of the charge exchange rate deduced from in situ measurements with the photoionization rate. Solar minimum values are in good agreement, but a clear discrepancy between the SWAN values and the in situ values appears after 1999.

of the electron impact ionization rate based on measurements of the electron distribution in the inner heliosphere.

[81] Third, the model used to fit the data is based on the classical hot model. This model assumes that at a large distance from the Sun, the hydrogen gas is well represented by a Maxwell-Boltzman distribution. However, recent models [Izmodenov et al., 2001; Quémerais and Izmodenov, 2002] have shown that the distribution of hydrogen after crossing the heliospheric interface is better represented by a multicomponent gas, each component with a different set of parameters at large distance from the Sun. The two main components, representing roughly half of the total atoms, are a flow of unperturbed hydrogen atoms which crossed the interface without interaction with the protons, and a secondary, decelerated and heated, flow produced by charge exchange with the interstellar plasma. It is this second component that may hold the key to our current problem. Indeed, according to Izmodenov et al. [2001], the deceleration of the secondary component is of the order of $10 \mathrm{~km} / \mathrm{s}$. This smaller bulk velocity in the solar rest frame makes the secondary flow more sensitive to radiation pressure and ionization effects. A correct estimate of this phenomenon requires modifying our current modeling of the hydrogen distribution to take into account the various components of the hydrogen gas. Future developments will study the three hypotheses presented in this section to try to reconcile the SWAN results and the in situ measurements of the solar wind mass flux.

[82] Acknowledgments. SOHO is a mission of international cooperation between ESA and NASA. SWAN was financed in France by CNES with support from CNRS and in Finland by TEKES and the Finnish Meteorological Institute.

[83] Amitava Bhattacharjee thanks the reviewers for their assistance in evaluating this paper.

\section{References}

Ajello, J. M., A. I. Stewart, G. E. Thomas, and A. Graps (1987), Solar cycle study of interplanetary Lyman-alpha variations: Pioneer-Venus orbiter sky background results, Astrophys. J., 317, 964.

Baranov, V. B., and Y. G. Malama (1993), Model of the solar wind interaction with the local interstellar medium: Numerical solution of selfconsistent problem, J. Geophys. Res., 98, 15,157.

Bertaux, J. L., and J. E. Blamont (1993), Evidence for an extra-terrestrial Lyman-alpha emission, the interstellar wind, Astron. Astrophys., 11, 200.

Bertaux, J. L., R. Lallement, V. G. Kurt, and E. N. Mironova (1985), Characteristics of the local interstellar hydrogen determined from Prognoz 5 and 6 interplanetary Lyman- $\alpha$ line profiles measurements with a hydrogen absorption cell, Astron. Astrophys., 150, 82.

Bertaux, J. L., et al. (1995), SWAN: A study of solar wind anisotropies on SOHO with Lyman alpha sky mapping, Sol. Phys., 162, 403.

Bertaux, J. L., R. Lallement, and E. Quemerais (1996), UV studies and the solar wind, Space Sci. Rev., 78, 317.

Bertaux, J. L., E. Quemerais, R. Lallement, E. Kyroela, W. Schmidt, T. Summanen, J. P. Goutail, M. Berthe, J. Costa, and T. Holzer (1997), First results from SWAN Lyman a solar wind mapper on SOHO, Sol. Phys., 175, 737.

Bertaux, J.-L., E. Quemerais, R. Lallement, E. Lamassoure, W. Schmidt, and E. Kyrölä (2000), Monitoring solar activity on the far side of the Sun from sky reflected Lyman radiation, Geophys. Res. Lett., 27, 1331.

Bzowski, M., T. Mkinen, E. Kyrl, T. Summanen, and E. Qumerais (2003), Latitudinal structure and north-south asymmetry of the solar wind from Lyman-alpha remote sensing by SWAN, Astron. Astrophys., 408, 1165, doi:10.1051/0004-6361:20031022.

Clarke, J. T., R. Lallement, J.-L. Bertaux, H. Fahr, E. Quemerais, and H. Scherer (1998), HST/GHRS observations of the velocity structure of interplanetary hydrogen, Astrophys. J., 499, 482.

de Toma, G., E. Quemerais, and B. R. Sandel (1996), Long-term variation of the interplanetary H Ly alpha glow: Voyager measurements and implications for the solar H Ly alpha irradiance, Astrophys. J., 491, 980. 
de Toma, G., O. R. White, B. G. Knapp, G. J. Rottman, and T. N. Woods (1997), Mg II core-to-wing index: Comparison of SBUV2 and SOLSTICE time series, J. Geophys. Res., 102, 2597.

Gloeckler, G., and J. Geiss (2004), Composition of the local interstellar medium as diagnosed with pickup ions, Adv. Space Res., 34, 5360.

Hall, D. T., D. E. Shemansky, D. L. Judge, P. Gangopadhyay, and M. A. Gruntman (1993), Heliospheric hydrogen beyond 15 AU: Evidence for a termination shock, J. Geophys. Res., 98, 15,185.

Izmodenov, V. V., M. Gruntman, and Y. G. Malama (2001), Interstellar hydrogen atom distribution function in the outer heliosphere, J. Geophys. Res., 106, 10,681.

Joselyn, J. A., and T. E. Holzer (1975), The effect of asymmetric solar wind on the Lyman alpha sky background, J. Geophys. Res., 80, 903.

Keller, H. U., K. Richter, and G. E. Thomas (1981), Multiple scattering of solar resonance radiation in the nearby interstellar medium. II, Astron. Astrophys., 102, 415.

Koutroumpa, D., R. Lallement, J. L. Bertaux, E. Quémerais, and S. Ferron (2005), SOHO/SWAN hydrogen cell data analysis, in Solar Wind 11, edited by B. Fleck and T. H. Zurbuchen, Eur. Space Agency Spec. Publ., ESA SP-592, 163.

Kumar, S., and A. L. Broadfoot (1979), Signatures of solar wind latitudinal structure in interplanetary Lyman-alpha emissions: Mariner 10 observations, Astrophys. J., 228, 302.

Kyrölä, E., T. Summanen, W. Schmidt, T. Mäkinen, E. Quemerais, J.-L. Bertaux, R. Lallement, and J. Costa (1998), Preliminary retrieval of solar wind latitude distribution from Solar Wind Anisotropies/SOHO observations, J. Geophys. Res., 103, 14,523-14,538.

Lallement, R., J. L. Bertaux, and V. G. Kurt (1985), Solar wind decrease at high heliographic latitudes detected from Prognoz interplanetary Lyman alpha mapping, J. Geophys. Res., 90, 1413.

Lallement, R., J. C. Raymond, J.-L. Bertaux, E. Qumerais, Y.-K. Ko, M. Uzzo, D. McMullin, and D. Rucinski (2004), Solar cycle dependence of the helium focusing cone from SOHO/UVCS observations: Electron impact rates and associated pickup ions, Astron. Astrophys., 426, 867.

Lallement, R., E. Quémerais, J. L. Bertaux, S. Ferron, D. Koutroumpa, and R. Pellinen (2005), Deflection of the interstellar neutral hydrogen flow across the heliospheric interface, Science, 307, 1447

Lemaire, P., C. Emerich, J.-C. Vial, W. Curdt, U. Schhle, and K. Wilhelm (2002), Variation of the full Sun hydrogen Lyman a and profiles with the activity cycle, in Proceedings of the SOHO 11 Symposium, edited by A. Wilson, Eur. Space Agency Spec. Publ., ESA SP-508, 219.

McComas, D. J., H. O. Funsten, J. T. Gosling, and W. R. Pryor (1999), Ulysses measurements of variations in the solar wind-interstellar hydrogen charge exchange rate, Geophys. Res. Lett., 26, 2701.

Ogawa, H. S., C. Y. R. Wu, P. Gangopadhyay, and D. L. Judge (1995), Solar photoionization as a loss mechanism of neutral interstellar hydrogen in interplanetary space, J. Geophys. Res., 100, 3455-3462.
Press, W. H., B. P. Flannery, and S. A. Teukolsky (1986), Numerical Recipes: The Art of Scientific Computing, Cambridge Univ. Press, New York.

Pryor, W. R., C. A. Barth, C. W. Hord, A. I. F. Stewart, K. E. Simmons, J. J Gebben, W. E. McClintock, S. Lineaweaver, J. M. Ajello, and W. K. Tobiska (1996), Latitude variations in interplanetary Lyman-a data from the Galileo EUVS modeled with solar He $1083 \mathrm{~nm}$ images, Geophys. Res. Lett., 23, 1893.

Pryor, W. R., J. M. Ajello, D. J. McComas, M. Witte, and W. K. Tobiska (2003), Hydrogen atom lifetimes in the three-dimensional heliosphere over the solar cycle, J. Geophys. Res., 108(A10), 8034, doi:10.1029/ 2003JA009878.

Quémerais, E. (2000), Angle dependent partial frequency redistribution in the interplanetary medium at Lyman alpha, Astron. Astrophys., 358, 353. Quémerais, E., and J. L. Bertaux (1993), Radiative transfer in the interplanetary medium at Lyman alpha, Astron. Astrophys., 277, 283.

Quémerais, E., and J.-L. Bertaux (2002), 14-day forecast of solar indices using interplanetary Lyman a background data, Geophys. Res. Lett., 29(2), 1018, doi:10.1029/2001GL013920.

Quémerais, E., and V. Izmodenov (2002), Effects of the heliospheric interface on the interplanetary Lyman alpha glow seen at 1 AU from the Sun, Astron. Astrophys., 396, 269.

Quémerais, E., J. L. Bertaux, B. R. Sandel, and R. Lallement (1994), A new measurement of the interplanetary hydrogen density with ALAE/ATLAS 1, Astron. Astrophys., 290, 941.

Summanen, T., R. Lallement, and E. Qumerais (1997), Solar wind proton flux latitudinal variations: Comparison between Ulysses in situ data and indirect measurements from interstellar Lyman a mapping, J. Geophys. Res., 102, 7051 .

Thomas, G. E. (1978), The interstellar wind and its influence on the interplanetary environment, Annu. Rev. Earth Planet. Sci., 6, 173.

Thomas, G. E., and R. F. Krassa (1971), OGO-5 measurements of the Lyman alpha sky background, Astron. Astrophys., 11, 218.

Witt, N., P. W. Blum, and J. M. Ajello (1979), Solar wind latitudinal variations deduced from Mariner 10 interplanetary $\mathrm{H} / 1216 \mathrm{~A} /$ observations, Astron. Astrophys., 73, 272.

Woods, T. N., W. K. Tobiska, G. J. Rottman, and J. R. Worden (2000), Improved solar Lyman a irradiance modeling from 1947 through 1999 based on UARS observations, J. Geophys. Res., 105, 27,195.

J.-L. Bertaux, S. Ferron, D. Koutroumpa, R. Lallement, and E. Quémerais, Service d'Aéronomie, BP3, F-91 371, Verrières le Buisson, France. (eric.quemerais@aerov.jussieu.fr)

E. Kyrölä and W. Schmidt, Finnish Meteorological Institute, P.O. Box 503, Helsinki FIN-00101, Finland. 